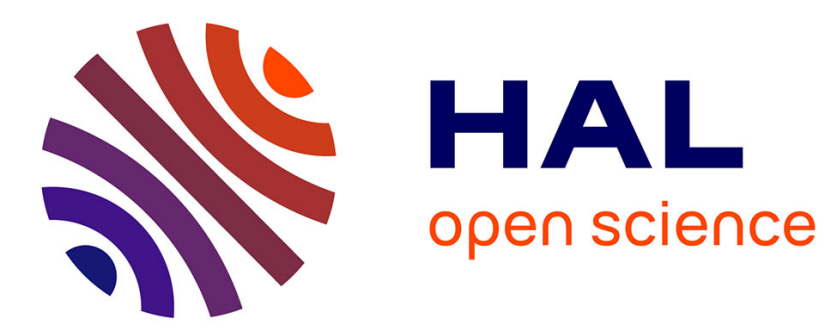

\title{
Robot anguille sous-marin en 3D
}

Frédéric Boyer, Mazen Alamir, Damien Chablat, Wisama Khalil, Alban

Leroyer, Philippe Lemoine

\section{To cite this version:}

Frédéric Boyer, Mazen Alamir, Damien Chablat, Wisama Khalil, Alban Leroyer, et al.. Robot anguille sous-marin en 3D. Techniques de l'Ingénieur, Edition Techniques de l'Ingénieur, pp.S 7 856: 1-16, 2006. hal-00186672

\section{HAL Id: hal-00186672 \\ https://hal.science/hal-00186672}

Submitted on 11 Nov 2007

HAL is a multi-disciplinary open access archive for the deposit and dissemination of scientific research documents, whether they are published or not. The documents may come from teaching and research institutions in France or abroad, or from public or private research centers.
L'archive ouverte pluridisciplinaire HAL, est destinée au dépôt et à la diffusion de documents scientifiques de niveau recherche, publiés ou non, émanant des établissements d'enseignement et de recherche français ou étrangers, des laboratoires publics ou privés. 


\title{
ROBOT ANGUILLE
}

\author{
F. Boyer, M. Alamir, D. Chablat, W. Khalil, A. Leroyer, Ph. Lemoine \\ IRCCyN, UMR 6597
}

Ecole des Mines de Nantes

1 Rue de la Noë, B.P 92101

44321 Nantes cedex 3, FRANCE

email: Frederic.Boyer@emn.fr

Résumé : L'objectif de ce projet soutenu par le programme ROBEA CNRS est de concevoir, étudier et réaliser un robot "anguille » capable de nager en trois dimensions. Pour cela nous étudions sur la base de modèles continus macroscopiques, les problèmes de la simulation, locomotion et commande. L'étude s'appuie sur une analyse biomécanique de la nage et se concrétise par la réalisation d'une plate-forme logicielle et d'un prototype. Pour atteindre ces objectifs un groupe pluridisciplinaire d'équipes et laboratoires a été formé :

- Muséum National d'Histoire Naturelle (MNHN) : Laboratoire d'Ichtyologie

- Laboratoire de Mécanique des Fluides (LMF) de Nantes : Divisions Modélisation Numérique (DMN) et Hydrodynamique Navale (DHN)

- IRCCyN : Equipes Robotique, Méthodes de Conception Mécanique, Systèmes Temps Réel

- LAG : Axe Commande et Observation

- LIRMM : Equipe Robotique sous-Marine

\section{Introduction}

Comparées à nos réalisations technologiques, les performances des poissons font rêver. Au nombre de celles-ci on compte leurs prodigieuses capacités d'accélération pouvant atteindre jusqu'à 20 fois la gravité, leur vitesse excédant les $70 \mathrm{~km} / \mathrm{h}$, leur extraordinaire manœuvrabilité : virage à $180^{\circ}$ sans ralentir et sur des rayons de courbure de l'ordre du dixième de leur longueur, tandis que les véhicules actuels doivent ralentir de moitié et prendre des rayons de courbures de l'ordre de 10 fois leur longueur. En terme d'efficacité, leur rendement est de l'ordre de 10 fois supérieur à ceux de nos meilleurs sous-marins...etc... Ces chiffres motivent à eux seuls les efforts actuels pour comprendre et reproduire les solutions des poissons sur nos systèmes robotiques. Dans ce domaine, relevant de la bio-mimétique, la première des difficultés rencontrées est décrite en ces termes:

«Reproduire les performances d'un poisson par simple imitation de sa forme et de sa fonction serait impossible car la mise au point d'un véhicule fléchissant de façon lisse et continue est au-delà des possibilités actuelles de la robotique. »

(M.S.Triantafyllou, An efficient swimming machine, Scientific American, Mars 1995)

Ainsi, le caractère continu des poissons constitue-t-il la difficulté essentielle de la recherche dans ce domaine. C'est l'objet de ce projet que de renforcer le biomimétisme en réalisant un prototype de robot anguille «plus continu » que ses 
homologues actuels. Pour cela, l'architecture mécanique du prototype est basée sur l'empilement en série de plates-formes parallèles gainées par un organe continu flexible jouant le rôle de la peau. La modélisation elle-même, s'appuie sur des modèles dynamiques dits « macro-continus » ( « macro » pour « macroscopiques ») basés sur la théorie des poutres Cosserats actionnées de manières continue.

Afin d'atteindre cet objectif nous avons débuté le projet par une étude bio-mécanique (§2). Sur la base de cette étude, la conception (\$3) assistée d'une modélisation macroscopique de type continu (macro-continue) résumée dans la section $\$ 4$ a été lancée et menée en parallèle d'une modélisation poly-articulée (\$5) plus fidèle à la réalité technologique du futur prototype. Enfin dès le départ une modélisation du contact fluide structure a été initiée (\$6). Soulignons que nous avons d'emblée adopté une approche hiérarchisée de modélisation tant pour la mécanique du robot que pour le contact fluide- structure. Sur la base de ces modèles et simulateurs associés, la commande est en cours d'étude (\$7) et sera implémentée in fine sur une architecture informatique abordée dans la section 8.

\section{La nage de l'anguille}

(Acteurs : F. Meunier (MNHN), tous les participants)

Dans la nature, on rencontre deux grands types de poissons chacun étant inféodé à un type de nage. Les premiers sont dits caranguiformes, ce sont les meilleurs nageurs en eau libre, tels le thon. Les seconds sont les anguilliformes, tels l'anguille ou la murène dont les capacités de manœuvrabilité atteignent des records. C'est ce second type de performance que notre projet se veut d'atteindre. Dans ce cas de figure, la manœuvrabilité est le résultat de la redondance élevée (hyper-redondance) induite par les déformations du corps du poisson relativement aux dimensions de la tâche (mouvoir sa tête). Avant toute investigation technique, le projet a démarré par une étude de la littérature bio-mécanicienne des poissons en général et de l'anguille en particulier. Chaque acteur du projet, selon ses préoccupations a pris en charge un domaine bibliographique. Les concepteurs ont étudié le système «squelette muscles - tendons », les acteurs de la modélisation et la commande ont étudié la biomécanique de la nage, sous l'aspect «mécanique des fluides » ou plus globalement, sous celui des allures de la nage. Concernant ce dernier point, les données des zoologistes expérimentateurs relatives aux allures de nage de l'anguille sont aujourd'hui restreintes à la locomotion plane et se présentent sous la forme de films dont sont extraites les lois d'évolutions en déplacement et orientation des vertèbres ainsi que celles des flexions intervertébrales prenant dans notre terminologie, le sens de courbure. A partir de ces données nous nous sommes attachés à caractériser et identifier des allures simplifiées sous-tendant des paramétrages minimaux des déformées mises en jeu. Nous avons pour cela mis à jour des lois de propagation d'ondes sinusoïdales de courbure progressives ou rétrogrades combinées à des courbures (de tangage et lacet). Afin de compenser les couplages induits par les courbures, des lois de torsion sont en cours d'étude. Sur la base de ces réflexions, la cinématique interne du robot a été décidée : il pourra fléchir dans les deux plans (lacet / tangage) et se tordre autour de sa colonne. Le prototype comprendra 12 vertèbres (soit 36 ddls internes mini), une tête rigide et une queue passive. La tête sera munie d'ailerons latéraux mimant les nageoires pectorales de l'animal dédiées au contrôle des roulis et tangage. 


\section{Conception du Prototype}

Acteurs : D. Chablat, P. Wenger, G. Branchu, P. Molina (IRCCyN)

\subsection{Introduction}

Depuis l'origine de la robotique, les ingénieurs ont constamment adapté leur conception aux technologies disponibles. Pour les robots, lorsque l'on parle de technologie, on entend surtout la technologie des actionneurs, de l'informatique ou des matériaux. Ainsi, en parcourant l'historique des catalogues de robots, on s'aperçoit que pour une même morphologie de robot, l'augmentation de la puissance des moteurs électriques ainsi que leur miniaturisation a permis dans un premier temps de remplacer les moteurs hydrauliques puis de simplifier et réduire le nombre de pièces par l'élimination des parallélogrammes ou des masses d'équilibrage. Dans un autre ordre d'idée, l'augmentation de la puissance des moyens informatiques a permis d'imaginer des structures mécaniques plus complexes et l'intégration de modèles dynamiques. Ainsi, à partir des structures mécaniques simples telles que des robots cartésiens ou anthropomorphes, on a vu apparaître des robots à structures mécaniques dites parallèles telles que les plate-formes de Gough-Stewart [1].

Pour la conception d'un robot anguille, nous devons adapter nos contraintes à des technologies matures. En effet, nous aurions pu utiliser des actionneurs à mémoire de forme ou des piézoélectriques si ceux-ci avaient possédé la dynamique et la puissance demandées pour le déplacement d'un robot dans l'eau. Aussi, nos choix se sont-ils orientés vers des micro-moteurs électriques à courant continu qui ont comme principal avantage de pouvoir être commandé en couple (à la différence des servomoteurs). Dans un même ordre d'idée, notre prototype étant unitaire, nous pouvons choisir des technologies qui auraient été inadaptées pour la grande série (principalement pour l'électronique).

\subsection{Choix de l'architecture mécanique}

À partir de l'étude biomécanique, il a été décidé de réaliser le prototype par l'empilement de 12 vertèbres ayant chacune 3 degrés de liberté de rotation. Pour notre étude, les contraintes suivantes ont été considérées :

- Réduire au maximum l'espace inter-vertébral afin de pouvoir s'approcher au maximum du modèle de déformation continue (Figure 1) ;

- Utiliser au maximum la section elliptique des vertèbres (Figure 1) ;

- Équilibrer le placement des éléments mécaniques afin d'assurer l'équilibre hydrostatique des vertèbres ;

- Trouver les mécanismes les plus robustes vis-à-vis des erreurs d'assemblage. 


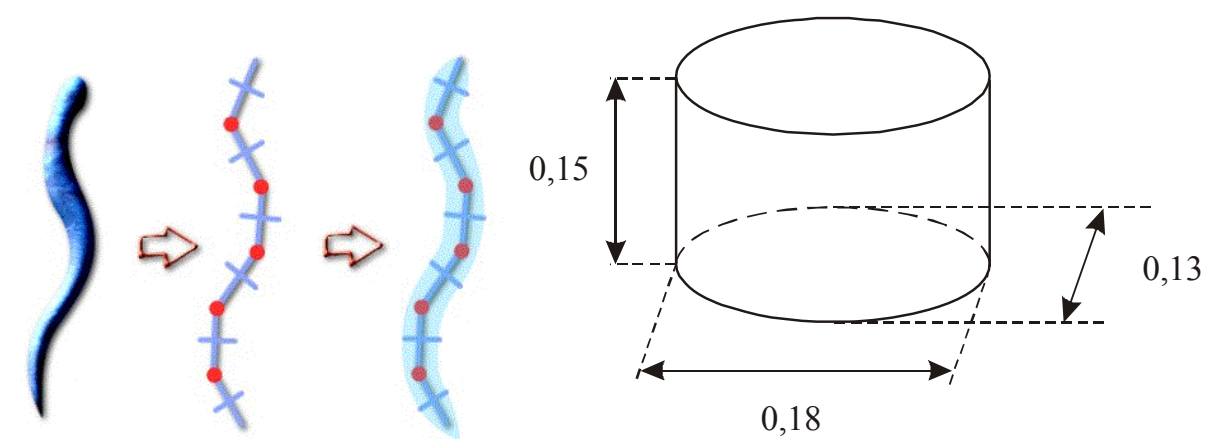

Figure 1 - Décomposition du corps de l'anguille en vertèbre avec leurs dimensions

Afin de pouvoir loger la mécanique, l'informatique et l'électronique dans le corps de l'anguille, nous avons fixé les dimensions suivantes pour chaque vertèbre: longueurs des focales $0,18 \mathrm{~m}$ et $0,13 \mathrm{~m}$, hauteur de $0,15 \mathrm{~m}$. Ceci revient à construire une anguille de plus de $2 \mathrm{~m}$ de long lorsque l'on tient compte de la tête et de la queue.

Sur la base d'une observation du système musculaire des poissons, on est tenté de réaliser nos vertèbres avec uniquement des actionneurs linéaires. Malheureusement, nous constatons que pour des petits encombrements, il existe peu d'alternatives robustes aux actionneurs rotatifs. En effet, pour réaliser une translation, la majeure partie des actionneurs linéaires utilise un actionneur rotatif, couplé à une liaison hélicoïdale. Les pertes dues aux frottements sont, dans ce cas, non négligeables. De plus, deux inconvénients s'ajoutent à ce type d'actionnement :

- L'encombrement : moteur + guidage ;

- Les débattements réduits

De même, la réalisation des vertèbres à partir d'une architecture cinématique sérielle a été écartée. En effet, l'utilisation d'une chaîne cinématique sérielle telle que représentée dans la figure 2 pose les problèmes suivants :

- Les moteurs sont placés de manière asymétrique ;

- Le couplage entre les moteurs (1) et (3) de chaque vertèbre nécessite la présence d'un montage complexe pour le transfert des contraintes entre les vertèbres ;

- Le déplacement du moteur (2) entraîne des déplacements de masses importantes.

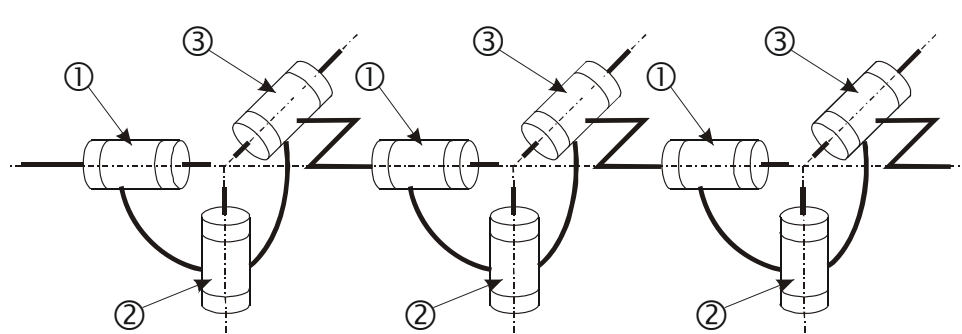

Figure 2 - $\quad$ Prototype de robot anguille basé sur une architecture complètement sérielle

Finalement, nous avons opté pour une architecture parallèle. Ce choix fait, il existe de nombreuses solutions parallèles réalisant un poignet sphérique. Elles sont 
habituellement classifiées suivant les propriétés suivantes [2]:

- symétrique / asymétrique

- isostatique / hyperstatique

- actionneurs linéaires / actionneurs rotatifs.

Cependant, et malgré les efforts de classifications, peu de réalisations technologiques de «liaison rotule parallèle » existent à ce jour. Parmi, ces rares prototypes, le plus connu en robotique est probablement l'œil agile développé par Clément Gosselin [3]. Cette architecture a été utilisée pour orienter une caméra dans l'espace (d'où son nom d'œil agile) ou comme périphérique haptique [4]. Elle est constituée de trois moteurs rotatifs dont les axes se croisent au centre de la rotule et de trois « pattes » constituées de deux pivots chacune, dont les axes coupent aussi le centre de la rotule (Figure 3). Ce sont ces pattes qui réalisent la liaison entre la partie fixe du mécanisme et la caméra (respectivement le poigné de l'utilisateur).
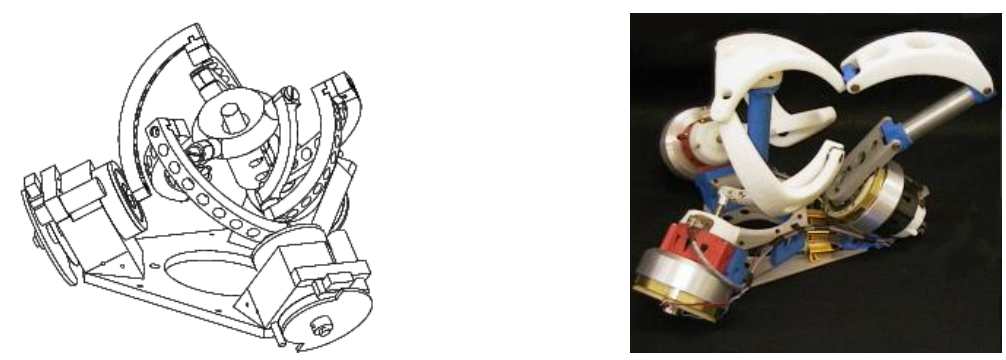

Figure 3 - $\quad$ L'œil agile et le ShaDe développés à l'Université Laval de Québec

Enfin, lorsque l'on assemble en série un tel mécanisme répété à l'identique, tous les efforts transitent par l'intermédiaire de chaque moteur, obligeant à renforcer les liaisons pivots de ces derniers. Pour éliminer ce problème, nous avons étudié une famille de poignet possédant une rotule passive au centre de la rotation (Figure 4).
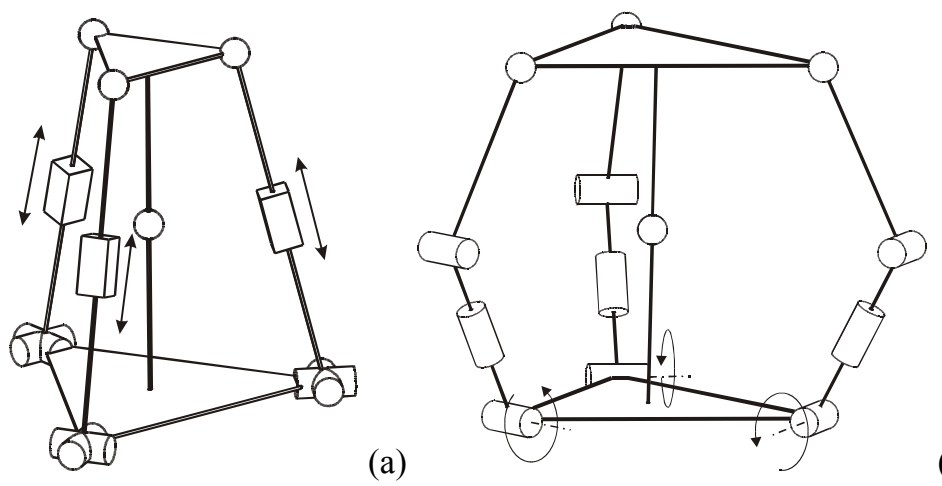

(b)

Figure 4 - Exemple de poignet sphérique avec le centre de rotation contraint par une articulation passive avec (a) actionneur linéaire et (b) actionneur rotatif

Ainsi, une solution entre l'œil agile et un poignet parallèle à 4 pattes peut être obtenue en motorisant la patte portant la rotule. Dans ce cas, on obtient une patte centrale constituée d'une pivot motorisée suivi d'un cardan. En affectant les 2 autres pattes au contrôle des deux rotations du cardan, on obtient le poignet représenté dans la figure 5 . Ce mécanisme est dérivé du mécanisme de poignet définis par Agrawal [5] avec des actionneurs rotatifs en lieu et place des actionneurs linéaires. 


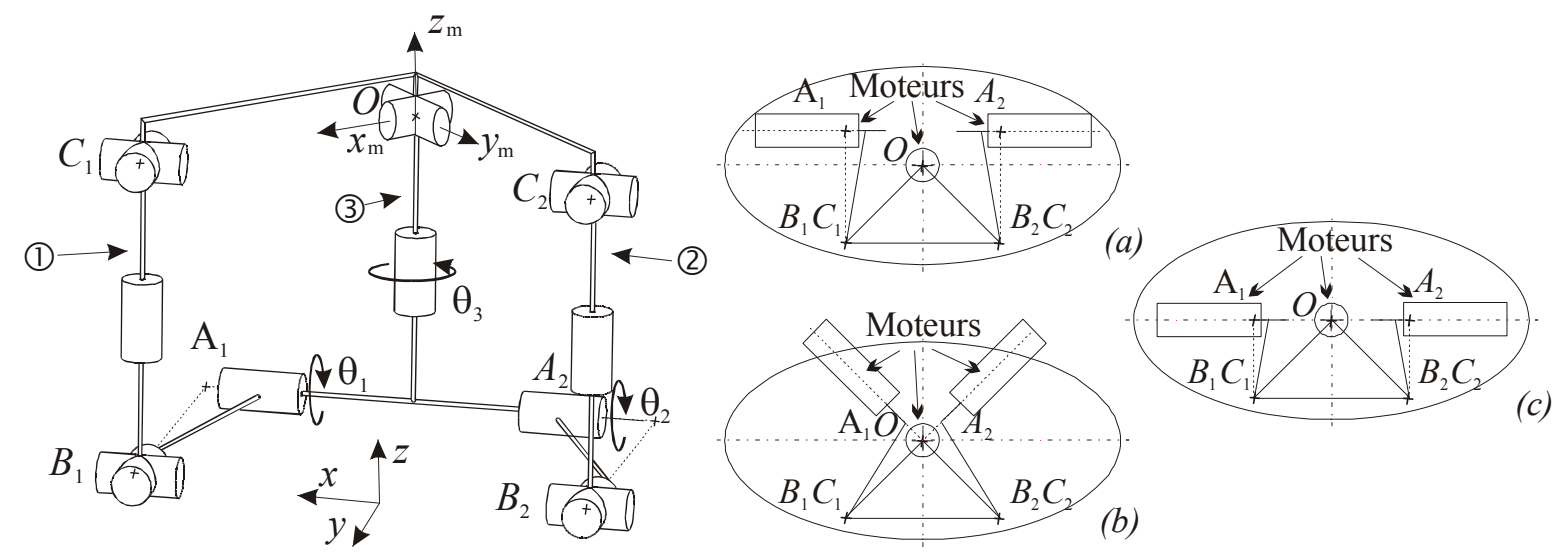

Figure 5 - Modélisation des vertèbres basée sur une architecture parallèle et son placement dans l'enveloppe elliptique

C'est cette architecture qui a été retenue. Aux nombres de ses avantages remarquons qu'elle est très compacte et peut facilement être placée dans la forme elliptique de l'anguille. De plus, la cinématique permet de reproduire, au travers de l'action des deux biellettes (1) et (2), le rôle des muscles attachés au squelette et travaillant en addition (dans le sens du lacet, pour la propulsion) et en soustraction (dans celui du tangage, pour la plongée). Le moteur placé sur la patte (3) permet de réaliser le mouvement de roulis. Plusieurs variantes de placement des moteurs ont été testées dans le projet (Figures 5 (a), (b) et (c)). C'est la solution (c) qui a été retenue car dans ce cas, l'axe des moteurs est colinéaire à l'axe de la plus grande focale de l'ellipse. Dans ce cas, lorsque l'angle de roulis est nul, les deux moteurs coaxiaux fonctionnent comme un différentiel. Sur la base de ce choix, les modèles cinématique et géométrique (direct et inverse) de ce robot parallèle ont été élaborés. Pour la commande, seul le modèle géométrique inverse est calculé. Avec notre conception, il s'écrit sous forme d'équations quadratiques, ce qui permet de le résoudre algébriquement. Cet aspect étant crucial en raison des puissances de calcul limitées des calculateurs embarqués. Pour éviter que ce moteur ne supporte des efforts axiaux trop importants, nous avons placé deux engrenages parallèles déportant le moteur relativement à l'axe vertébral. Ce dispositif permet aussi de placer un moteur dont la longueur axiale est importante.

\subsection{Assemblage des vertèbres}

Le corps de l'anguille est réalisé par la mise en série de ses vertèbres. Dans cet assemblage, l'emplacement des moteurs, de l'informatique embarquée et l'électronique de puissance doivent être pris en compte. Après analyse des besoins de calcul en ligne, le choix a été adopté d'affecter un micro-contrôleur à deux vertèbres. Les moteurs contrôlant le tangage et le lacet sont côte à côte tandis que ceux contrôlant le roulis sont en opposition. Ce choix permet d'équilibrer les masses sur deux vertèbres. Enfin, sur chaque section elliptique sera fixée la peau de notre robot. La figure 6 représente la position des différents éléments dans le prototype. 


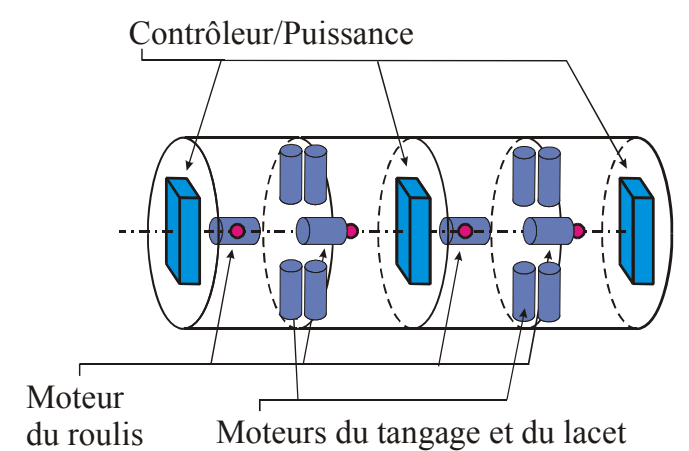

Figure 6 - $\quad$ Organisation des éléments mécaniques, de Figure 7 l'informatique et de l'électronique de puissance

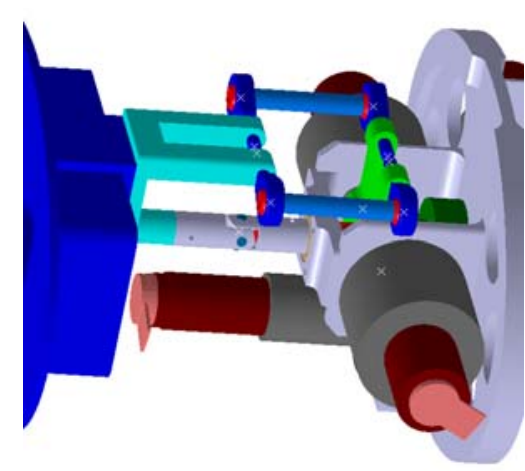

Modélisation CATIA

La modélisation sous CATIA de chaque vertèbre a permis de simuler le déplacement des moteurs et d'éviter les interférences entre les pièces en mouvement (Figure 7).

\subsection{Conception de la peau}

La peau de l'anguille sera fixée sur chaque vertèbre, l'objectif de notre conception étant d'assurer le maintien de cet organe afin de réaliser un contact continu. La difficulté résulte ici de la tension entre deux objectifs contradictoires mettant tous deux en jeux les déformations de l'organe. En effet, la peau doit à la fois offrir une déformation très aisée en flexion-flexion-torsion tout en garantissant une résistance importante aux pressions du fluide. A cette difficulté de principe, s'ajoute les déformations importantes que doit subir l'organe. En témoigne la figure 8 relative au plan du lacet. Lorsque l'angle de lacet est égal à 30 degrés, la courbure extérieure augmente de $24 \%$ tandis que la courbure intérieure diminue de $28 \%$. Afin d'éviter que la peau ne plisse, une première solution serait de la pré-contraindre lorsque la courbure extérieure est minimale. Malheureusement, si la peau est constituée d'un matériau élastique (caoutchouc, lycra), son étirement axial, s'accompagne d'un inévitable rétrécissement transverse (fig.9).
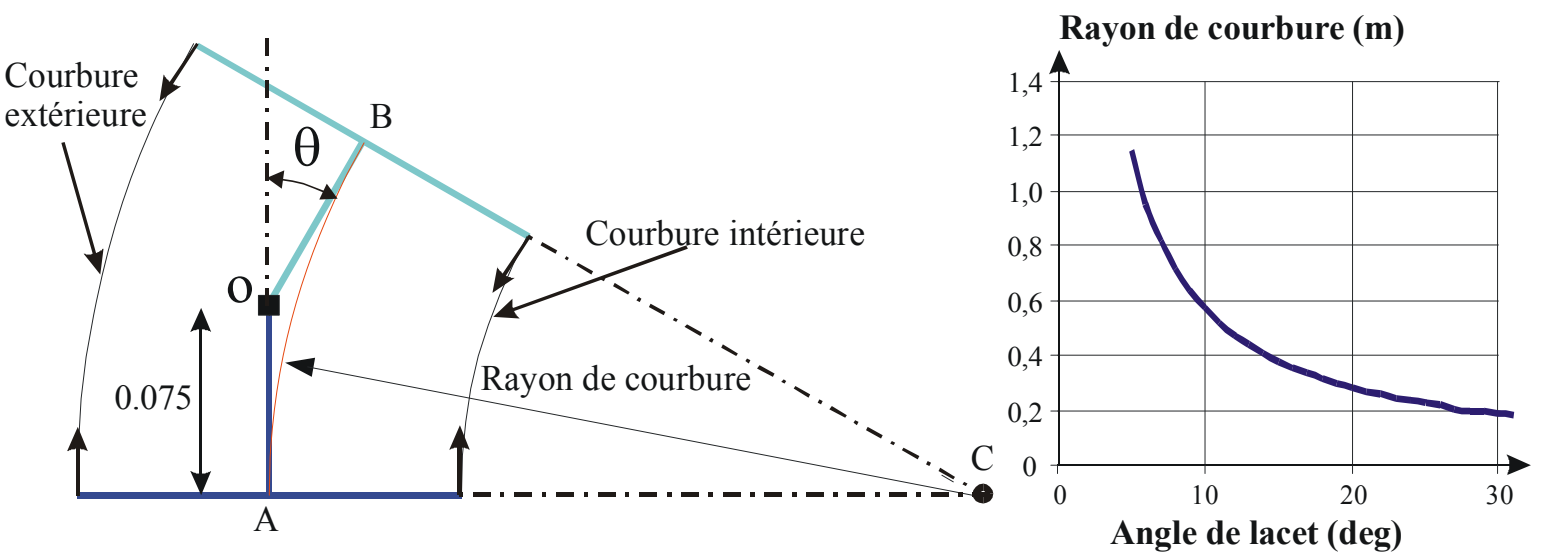

Figure 8 - $\quad$ Lien entre le rayon de courbure de l'anguille et l'angle de lacet

Aussi, le choix a t'il été adopté de soutenir une telle peau d'une structure sousjacente. Cette structure pourrait par exemple être basée sur l'empilement de vertèbre intermédiaires évidées connectées par des anneaux en caoutchouc (fig. 9). Au nombre des avantages d'une telle solution, on compte outre la garantie de continuité en courbure recherchée: les grands débattements octroyés, la facilité de montage, 
et son faible encombrement radial.
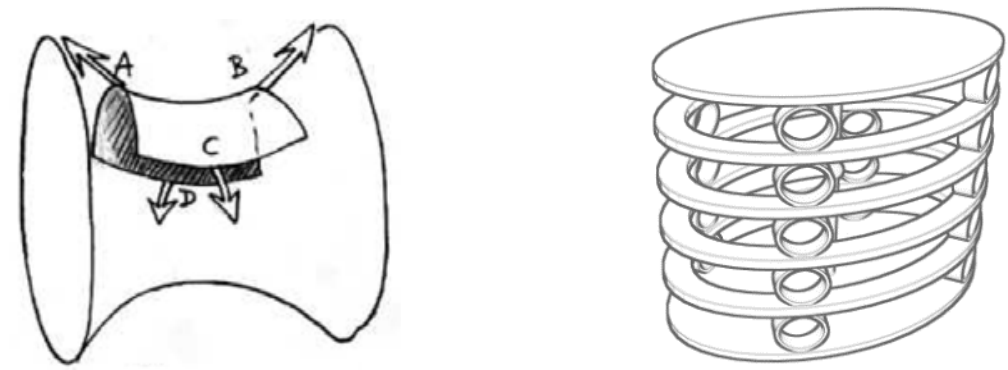

Figure 9 - Déformation de la peau sans armature et armature proposée

\section{Modélisation du prototype}

Acteurs : F. Boyer, W. Khalil, G. Levey, M. Porez, G. Gallot (IRCCyN)

\subsection{Introduction}

La modélisation du projet a pour objets essentiels : $1^{\circ}$ ) d'orienter et dimensionner la conception du prototype, $2^{\circ}$ ) de mettre à jour des modèles simples dédiés à la commande, $3^{\circ}$ ) et de manière annexe, de produire des connaissances générales sur la locomotion des poissons. Elle se scinde en deux parties: le «solide » et le «fluide ». Chacun de ces deux volets est ensuite décliné en trois modèles hiérarchiques du « macro » au « micro ». Cette approche hiérarchisée nous permet de maîtriser la modélisation du niveau le plus fin au plus grossier et de faire remonter et/ou descendre les enseignements acquis par un niveau à tous les autres ; avec en ligne de mire, la conception du prototype et sa commande. Concernant le volet « solide », la modélisation du prototype se décline comme suit:

$\left.1^{\circ}\right)$ la modélisation macro-continue

$2^{\circ}$ ) la modélisation poly-articulée sérielle

$3^{\circ}$ ) la modélisation poly-articulée hybride

Le premier de ces niveaux est dédié à l'étude des allures de nages et à la commande. II est calé sur l'animal et réalise ainsi la référence du « prototype idéal » Quant au second il a pour intérêt essentiel de «faire le pont» entre le premier niveau et le dernier qui réalise le « modèle technologique » du prototype.

\subsection{Modélisation macro-continue}

Lorsque son nombre de degrés de liberté internes est très élevé (ce qui est le cas de notre futur prototype), il devient pertinent de modéliser un système poly-articulé de manière continue. L'approche ici poursuivie s'appuie sur la théorie des poutres Cosserat [6]. Sous cette hypothèse, la poutre est réalisée par l'empilement continu de sections rigides connectées par des liaison infinitésimales prenant le sens d'une "cinématique de poutre ». Cette approche est, nous le pensons, une voie prometteuse pour l'étude de la dynamique des robots hyper-redondants. Ainsi, si l'on considère ces derniers comme réalisés par l'empilement sériel de structures 
parallèles (c'est notre choix), à chaque architecture parallèle va correspondre une cinématique de poutre. Certaines, telle la plate-forme rotule, ou de Gough-Stewart ont directement leur équivalent continu dans la théorie des poutres de Kirchoff ou celle de Timoshenko, tandis que d'autres ne correspondent au contraire à aucune théorie de poutre connue. Quoiqu'il en soit un tel rapprochement est toujours possible. Dans le cas du robot anguille, la cinématique intervertébrale est de type rotule et la théorie de poutre associée est celle des poutres de Kirchoff inextensibles. L'avantage de cette approche, comparée aux solutions existantes basées essentiellement sur les "back-bone curves » [7] est que dans le contexte des poutres, l'analogie entre les systèmes poly-articulés et continus se poursuit pas à pas, de la géométrie à la dynamique. Ainsi, les vertèbres deviennent les sections, les déformations (courbures, torsion, extension...) tiennent lieu de coordonnées articulaires intervertébrales...etc. Sous ces hypothèses, le paramétrage de l'anguille ou « prototype idéal » est récapitulé dans la figure 10.

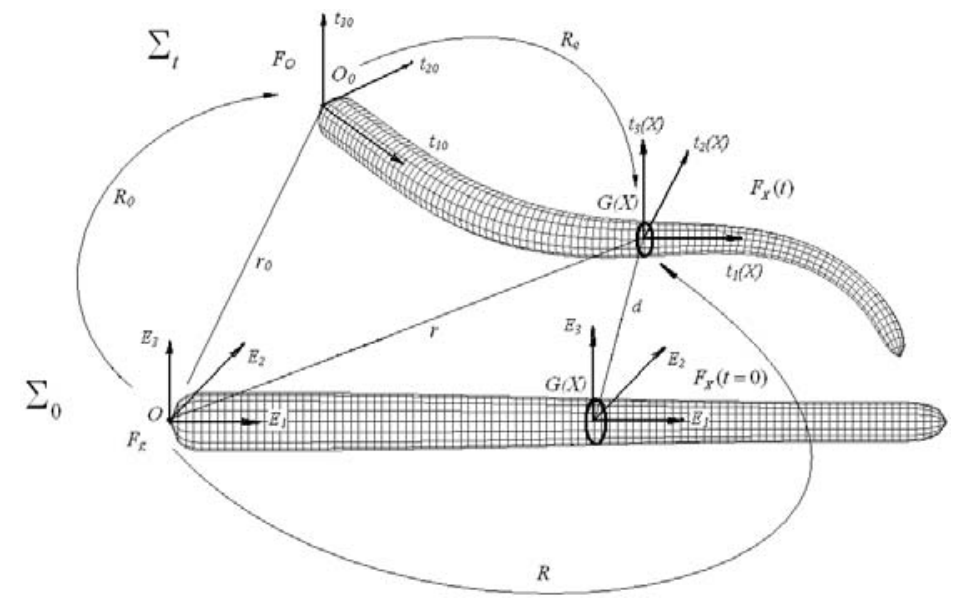

Figure 10 - Paramétrage continu de l'anguille

\subsection{Modèle dynamique macro continu}

Dans le cadre du projet, nous avons donc dans un premier temps établi le modèle continu de notre futur prototype que nous avons nommé « macro-continu». Sur la base d'une approche de type " géométriquement exacte » des milieux continus telle que proposée par J.C. Simo pour l'étude numérique des milieux Cosserat [8], nous avons établi les équations de la dynamique du robot continu via un calcul des variations sur le groupe de Lie $S E(3)$ de ses sections. Le modèle obtenu est un système d'équations aux dérivées partielles réglant la dynamique du robot sur son espace des configurations ici assimilé à un espace de courbes (paramétrées par l'abscisse matérielle de la poutre) dans le groupe de Lie $S E(3)$ des déplacements de ses sections. Ce jeu d'équations est rapporté ci dessous. Elle mettent en jeux des tenseurs dépendant de l'espace mais aussi du temps $t$ et l'on convient de noter par un point l'opérateur « $\partial . / \partial t »$ et par un « prime », l'opérateur « $\partial . / \partial X » .:$

- Équations d'équilibre (la longueur de l'anguille est normalisée):

$$
\forall X \in] 0,1\left[:\left(\begin{array}{c}
\rho A \ddot{r} \\
\rho I \dot{\omega}+\omega \times(\rho I \omega)
\end{array}\right)+\left(\begin{array}{c}
n^{\prime} \\
(R M)^{\prime}+t_{1} \times n
\end{array}\right)=\left(\begin{array}{c}
f_{\text {fluide }} \\
c_{\text {drag }}
\end{array}\right)\right.
$$


Où l'on a introduit les quantités suivantes, toutes relatives à une section « étiquetée » par son abscisse curviligne $X$ comptée en partant de la tête le long de la colonne vertébrale.

- $\quad \rho$ : masse volumique de l'anguille,

- $A, I$ : aire et tenseur d'inertie de la section de l'anguille

- $\omega, \dot{\omega}$ : vitesse et accélération angulaire de la section

- $\quad \ddot{r}$ : accélération linéaire de la section

- $n$ : résultante des forces internes appliquées à la section

- $t_{1}$ : normale unitaire à la section (tangente à la colonne)

- $f_{\text {fluide }}, c_{\text {fluide }}$ : force et moment exercé par le fluide sur la section (cf. § 5.1)

Enfin, tous ces tenseurs sont exprimés dans la base fixe du référentiel terrestre excepté le suivant, exprimée dans le repère mobile de la section :

- $\quad M$ : résultante des moments internes appliqués à la section (couples de commande)

Et lié au repère fixe par :

- $\quad R$ : l'opérateur de rotation de la section par rapport au repère fixe.

Ces équations doivent être complétées de 2 équations de contrainte: l'une traduisant la loi de courbure-torsion imposée par les liaisons intervertébrales, la seconde imposant l'inextensibilité de la colonne vertébrale.

- Équations de contrainte :

$$
K=K_{d}(t), \quad r^{\prime}-t_{1}=0
$$

Où $K_{d}$ est le vecteur de courbure-torsion désiré exprimé dans le repère mobile des sections. Finalement, les solutions de cette équation sont spécifiées par les conditions limites en tête et queue de l'anguille.

- Conditions aux limites :

$$
\left(\begin{array}{c}
n(0) \\
M(0)
\end{array}\right)=\left(\begin{array}{c}
f_{\text {fluide, }} \\
0
\end{array}\right),\left(\begin{array}{c}
n(1) \\
M(1)
\end{array}\right)=\left(\begin{array}{l}
0 \\
0
\end{array}\right)
$$

Où $f_{\text {fluide, }}$ modélise la résultante des efforts fluide exercée sur la tête de l'anguille, toutes les autres contributions étant supposées nulles.

\subsection{Algorithmes continus}

Dans leurs principes, les équations précédents suffisent à résoudre le problème direct de la simulation qui se résume ici à connaître les accélérations des sections à partir des couples intervertébraux et de l'état du robot. L'approche serait alors 
typiquement de type éléments finis "géométriquement exact 》 [9]. En fait c'est le problème inverse qui a réclamé notre attention. Ce second problème consiste à calculer, connaissant les accélérations intervertébrales et l'état du robot, les couples intervertébraux ainsi que les accélérations de la tête de l'anguille. Afin de résoudre ce problème, mieux adapté à la recherche d'allures que le premier, nous avons opté pour une solution totalement différente des habitudes de la mécanique non linéaire des structures. Pour appréhender cette solution, il suffit de réaliser que les e.d.p. de la poutre Cosserat sont en fait la version continue des équations de Newton-Euler d'un manipulateur poly-articulé sériel (cf. § 4.5). Ainsi, en complétant ces équations dynamiques des modèles géométrique et cinématique (en vitesse et accélération) on fabrique un jeux d'équations, pendant continu des modèles de Newton-Euler discrets. Ces modèles, traduisant la cinématique continue de l'anguille se présente comme suit :

Modèle géométrique

- En orientation :

$$
R^{\prime}=R \hat{K}_{d}, \quad R(0)=R_{o}
$$

- En position :

$$
r^{\prime}=R E_{1}, r(0)=r_{o}
$$

Modèle des vitesses

- En orientation :

$$
\omega^{\prime}=R \dot{K}_{d}, \quad \omega(0)=\omega_{o}
$$

- En position :

$$
\dot{r}^{\prime}=\omega \times r^{\prime}, \quad \dot{r}(0)=\dot{r}_{o}
$$

Modèle des accélérations

- En orientation :

$$
\dot{\omega}_{e}^{\prime}=R \ddot{K}_{d}+\omega \times R \dot{K}_{d}, \dot{\omega}_{e}(0)=0
$$

- En position :

$$
\gamma_{e}^{\prime}=\dot{\omega}_{e} \times r^{\prime}+\omega \times\left(\omega \times r^{\prime}\right), \gamma_{e}(0)=0
$$

Où les tenseurs indexés par " ${ }^{\circ}$ » sont relatifs à la tête $(X=0), \hat{K}_{d}$ désigne le tenseur anti-symétrique associé au vecteur de courbure-torsion désiré $K_{d}, E_{1}$ est le premier vecteur de la base fixe et $\dot{\omega}_{e}$ et $\gamma_{e}$ sont les accélérations des sections privées des contributions $\dot{\omega}_{o}, \gamma_{o}$ apportées par la tête. 
Reste qu'en plus du caractère continu de ces nouvelles équations, elles se distinguent de celles des manipulateurs discrets par le fait que la base est ici remplacée par un corps mobile : « la tête de l'anguille ». II s'en suit que la dynamique de la tête de l'anguille doit aussi être ajoutée à la formulation précédente afin de la rendre solvable. Pour établir cette dynamique, un calcul des variations est encore possible mais cette fois appliqué sur l'espace des configurations du robot identifié au fibré $S E(3) \times S$, où $S$ est l'espace des formes internes du robot ("Shape space"), ici réalisé par un espace de courbes (paramétrées par l'abscisse matériel de la poutre) dans l'algèbre de Lie se(3). Ces équations se présentent comme suit :

$$
I\left(\begin{array}{c}
\ddot{r}_{o} \\
\dot{\omega}_{o}
\end{array}\right)+\left(\begin{array}{c}
f_{e} \\
c_{e}
\end{array}\right)=\left(\begin{array}{l}
\bar{f}_{\text {fluide }} \\
\bar{c}_{\text {fluide }}
\end{array}\right)
$$

Où de gauche à droite figurent le tenseur d'inertie de l'anguille complète rapporté à son extrémité (côté tête), i.e. à $X=0$, le vecteur des accélérations linéaires et angulaires de la tête, le vecteur des forces d'inerties (Coriolis - centrifuges et accélérations internes du corps), et enfin le vecteur des résultantes des forces et moments exercés par le fluide sur le corps et rapporté au point $X=0$ (§6.1).

Finalement, une fois ces trois jeux d'équations (e.d.p. sur le corps, dynamique de la tête, modèles cinématiques) en main, nous avons appliqué une approche de type Newton-Euler afin de résoudre le problème inverse. L'algorithme proposé n'est ni plus ni moins qu'une extension de l'algorithme de Luh-Walker et Paul aux robots continus avec base mobile [10]. Outre ce dernier aspect (aussi partagé par les modèles poly-articulés, c.f. ci après), l'algorithme se distingue de sa version discrète par le fait que les récurrences arrières (sur les efforts) et avant (sur les quantités cinématiques) sont remplacées par des o.d.e. en espaces intégrées à chaque pas de temps de la simulation.

\subsection{Résultats de la simulation}

Grâce à l'algorithme inverse macro-continu plusieurs allures 3D ont pu être étudiées. En combinant les courbures de tangage et de lacet on peut par exemple fabriquer des plongées en spirale (Figure 11) ou «droites » (Figure 12). Dans ces essais, le modèle du contact est de type masse (inerties) ajoutées et forces - couples de traînée (§6.1).

Figure 11 - Plongée en spirale
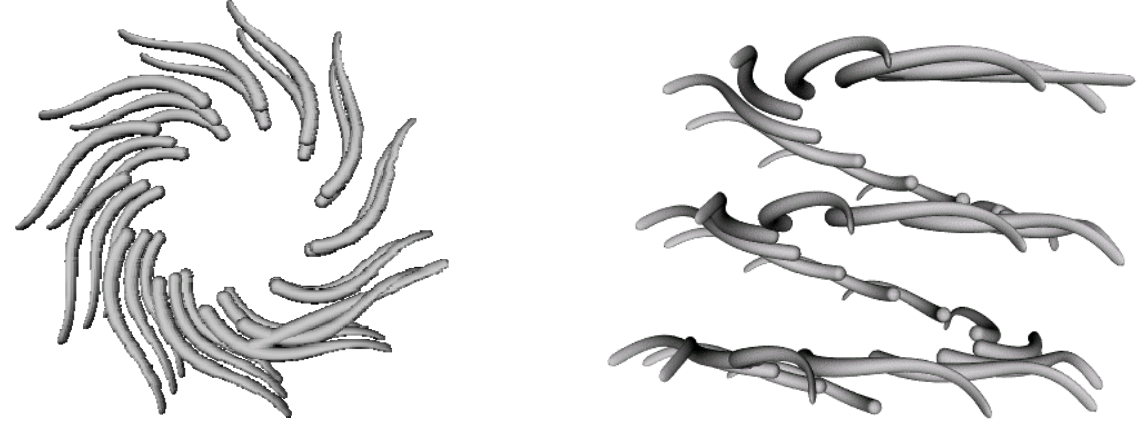


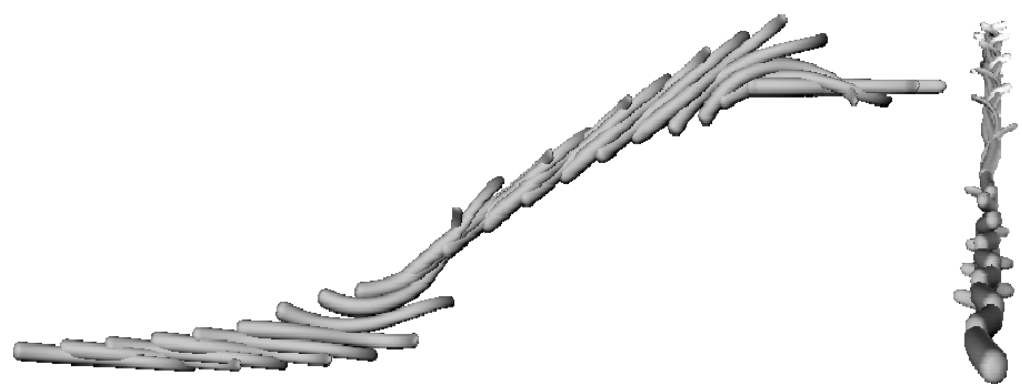

Figure 12 - Plongée droite

\subsection{Modèle poly-articulé}

La seconde et la troisième étape de la modélisation mécanique du robot considèrent ce dernier comme un système poly-articulé. Ainsi, passe t'on de la modélisation continue du poisson ou «prototype idéal », à celle de la réalité technologique du prototype hybride (sériel/parallèle), via un modèle simplifié de type sériel. C'est ce second modèle que nous allons à présent détailler.

\subsubsection{Modèle poly-articulé sériel}

Dans cette optique nous considérons que le robot anguille est composé de $n$ corps rigides connectés en série par des articulations de type rotule. Chaque articulation a été modélisée par trois articulations rotoïdes d'axes concourants, les deux premières articulations supportent des corps fictifs avec masses et longueurs nulles. Le troisième corps est constitué d'un cylindre d'une section elliptique proche de la forme retenue pour le prototype.

\subsubsection{La description géométrique du robot}

La description de la géométrie du robot est réalisée par les notations de Khalil et Kleinfinger [11] couramment utilisées pour décrire les robots manipulateurs, (figure 13). La structure est composée de $n+1$ corps notés $C_{o}, \ldots, C_{n}$ et de $n$ articulations. Le corps $C_{o}$ désigne la tête du robot et le corps $C_{n}$ représente sa queue. L'articulation $j$ connecte le corps $C_{j}$ au corps $C_{j-1}$. On définit un repère $R_{j}$ fixé au corps $C_{j}$;

Le repère $R_{j}$, est défini de sorte que :

- $\quad$ l'axe $z_{j}$ est porté par l'axe de l'articulation $j$;

- $\quad$ l'axe $x_{j}$ est porté par la perpendiculaire commune aux axes $z_{j}$ et $z_{j+1}$; 


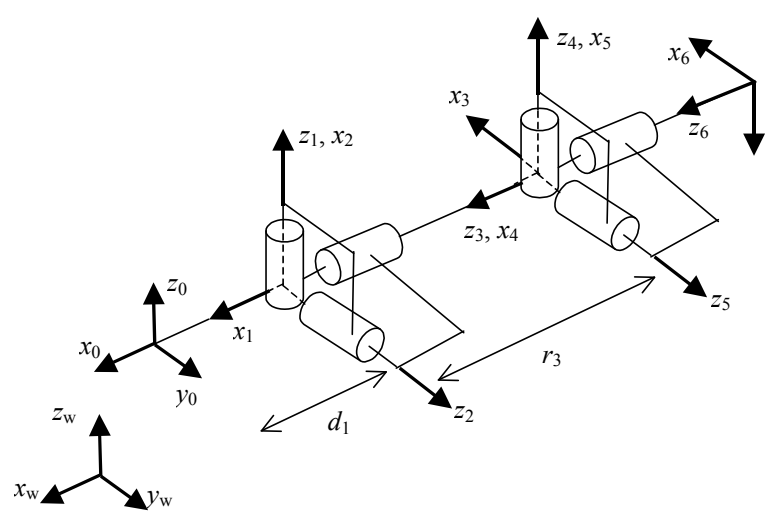

Figure 13 - Description géométrique de la structure serial de l'anguille

La matrice de transformation ${ }^{j-l} \boldsymbol{T}_{j}$ entre le repère $R_{j-1}$ et le repère $R_{j}$ s'exprime en fonction des paramètres suivants:

- $\alpha_{j}$ : l'angle entre $z_{j-1}$ et $z_{j}$ autour de $x_{j-1}$,

- $d_{j}$ : la distance entre $z_{j-1}$ et $z_{j}$ suivant $x_{j-1}$,

- $\theta_{j}$ : l'angle entre $x_{j-1}$ et $x_{j}$ autour de $z_{j}$,

- $r_{j}$ : la distance entre $x_{j-1}$ et $x_{j}$ le long de $z_{j}$.

La variable de l'articulation $j$, est donnée par:

$$
\theta_{j}=q_{j}+q_{o j}
$$

Où $q_{o j}$ est un offset.

On obtient ${ }^{j-1} \boldsymbol{T}_{j}$ :

$$
{ }^{j-1} \boldsymbol{T}_{j}=\left[\begin{array}{cccc}
C \theta_{j} & S \theta_{j} & 0 & d_{j} \\
C \alpha_{j} S \theta_{j} & C \alpha_{j} C \theta_{j} & -S \alpha_{j} & -r_{j} S \alpha_{j} \\
S \alpha_{j} S \theta_{j} & S \alpha_{j} C \theta_{j} & C \alpha_{j} & r_{j} C \alpha_{j} \\
0 & 0 & 0 & 1
\end{array}\right]
$$

Les paramètres géométriques de l'anguille qui est composé de 12 modules, donc 36 articulations rotoïdes, sont donnés dans le tableau suivant 


\begin{tabular}{|c|c|c|c|c|c|}
\hline $\mathrm{j}$ & $\alpha_{\mathrm{j}}$ & $\mathrm{d}_{\mathrm{j}}$ & $\theta_{\mathrm{j}}$ & $\mathrm{r}_{\mathrm{j}}$ & $\mathrm{q}_{0 \mathrm{j}}$ \\
\hline 1 & 0 & 0.4 & $\theta_{1}$ & 0 & 0 \\
\hline 2 & $-\pi / 2$ & 0 & $\theta_{2}$ & 0 & $\pi / 2$ \\
\hline 3 & $-\pi / 2$ & 0 & $\theta_{3}$ & 0.14 & $\pi / 2$ \\
\hline 4 & $\pi / 2$ & 0 & $\theta_{4}$ & 0 & $-\pi / 2$ \\
\hline$\ldots$ & $\ldots$ & $\ldots$ & $\ldots$ & $\ldots$ & $\ldots$ \\
\hline 35 & $-\pi / 2$ & 0 & $\theta_{35}$ & 0 & $-\pi / 2$ \\
\hline 36 & $-\pi / 2$ & 0 & $\theta_{36}$ & 0.14 & $\pi / 2$ \\
\hline
\end{tabular}

Tableau 1 - Paramètres géométriques de l'Anguille

\subsubsection{Modèle dynamique inverse}

Concernant la modélisation dynamique nous avons développé les modèles dynamiques inverse et direct de cette structure pour la nage en 3D. Les modèles ont été obtenus par des algorithmes récursifs qui utilisent les équations de NewtonEuler. Dans la suite nous présentons l'algorithme du modèle dynamique inverse qui fournit les couples articulaires et les six composantes de l'accélération, de translation et rotation, de la tête en fonction des évolutions désirées de position, vitesses, accélérations des variables articulaires. Cet algorithme est facile à écrire et il est beaucoup plus simple à obtenir que toutes autres solutions lagrangiennes telles que préconisées par les chercheurs américains et japonais et dédiée à la nage plane.

L'algorithme du modèle dynamique inverse est fondé sur trois récurrences :

i) Récurrence avant pour $j=1, \ldots n$ :

Dans cette récurrence, on calcule les vitesses des corps par les relations suivantes:

$$
{ }^{j} T_{j-1}=\left[\begin{array}{cc}
{ }^{j} \boldsymbol{R}_{j-1} & -{ }^{j} \boldsymbol{R}_{j-1}{ }^{j-1} \hat{\boldsymbol{P}}_{j} \\
\mathbf{0}_{3 \times 3} & { }^{j} \boldsymbol{R}_{j-1}
\end{array}\right], \quad{ }^{j} V_{j}={ }^{j} T_{j-1}{ }^{j-1} V_{j-1}+\dot{q}_{j}{ }^{j} A_{j}
$$

Avec:

${ }^{j-1} \boldsymbol{R}_{j}$ : la matrice de rotation (3×3) du repère $R_{j}$ par rapport au repère $R_{j-1}$, ${ }^{j-1} \boldsymbol{P}_{j}$ : le vecteur (3x1) définissant la position de l'origine du repère $R_{j}$ dans $R_{j-1}$. ${ }^{j} V_{j}$ : le torseur cinématique du corps $j$, qui regroupe la vitesse de translation ${ }^{j} V_{j}$ et la vitesse de rotation ${ }^{j} \boldsymbol{\omega}_{j}$, toutes deux exprimées dans $R_{j}$ :

$$
V_{j}=\left[\begin{array}{ll}
\boldsymbol{V}_{\mathrm{j}}^{\mathrm{T}} & \boldsymbol{\omega}_{\mathrm{j}}^{\mathrm{T}}
\end{array}\right]^{\mathrm{T}}
$$

Et ${ }^{j} A_{j}$ est la matrice $(6 \times 1)$ définie par : 


$$
{ }^{j} A_{j}=\left[\begin{array}{c}
\mathbf{0}_{3 x 1} \\
{ }^{j} \boldsymbol{a}_{j}
\end{array}\right]
$$

Dans cette récurrence la vitesse de la tête est suppose connue.

On calcule également les éléments des accélérations et des efforts qui ne font pas intervenir les accélérations de la tête (inconnues), ce qui revient à calculer:

$$
{ }^{j} \boldsymbol{\gamma}_{j}=\left[\begin{array}{c}
{ }^{j} \boldsymbol{R}_{j-1}\left[{ }^{j-1} \boldsymbol{\omega}_{j-1} \times\left({ }^{j-1} \boldsymbol{\omega}_{j-1} \times{ }^{j-1} \boldsymbol{P}_{j}\right)\right] \\
{ }^{j} \boldsymbol{\omega}_{j-1} \times \dot{q}_{j}^{j} \boldsymbol{a}_{j}
\end{array}\right]+\ddot{q}_{j}{ }^{j} A_{j}
$$

et :

$$
{ }^{j} \boldsymbol{\beta}_{j}={ }^{j} F_{e j}+\left[\begin{array}{c}
{ }^{j} \boldsymbol{\omega}_{j} \times\left({ }^{j} \boldsymbol{\omega}_{j} \times{ }^{j} \mathbf{M S} \mathbf{S}_{j}\right) \\
{ }^{j} \boldsymbol{\omega}_{j} \times\left({ }^{j} \mathbf{J}_{j}{ }^{j} \boldsymbol{\omega}_{j}\right)
\end{array}\right]
$$

où l'on a introduit les notations:

${ }^{j} F_{e j}$ : forces extérieures exercées par le corps j sur l'environnement;

${ }^{j} \mathbf{J}_{j}$ : la matrice (3x3) d'inertie du corps $j$ dans $R_{j}$,

${ }^{j} \mathbf{M S}_{j}$ : Le vecteur (3x1) des premiers moments du corps $j$,

ii) Récurrence arrière pour $j=n-1, \ldots 0$ :

Dans cette récurrence, on calcule les éléments suivants qui permettent de calculer l'accélération de la tête :

$$
\begin{gathered}
{ }^{j} J_{j}^{+}={ }^{j} J_{j}+{ }^{j+1} T_{j}^{T}{ }^{j+1} J_{j+1}^{+}{ }^{j+1} T_{j} \\
{ }^{j} \boldsymbol{\beta}_{j}^{+}={ }^{j} \boldsymbol{\beta}_{j}+{ }^{j+1} T_{j}^{\mathrm{T}}{ }^{j+1} \boldsymbol{\beta}_{j+1}^{+}+{ }^{j+1} T_{j}^{\mathrm{T}}{ }^{j+1} J_{j+1}^{+}{ }^{j+1} \boldsymbol{\gamma}_{j+1}
\end{gathered}
$$

${ }^{j} J_{j}^{+}$est la matrice d'inertie spatiale $(6 \times 6)$ du corps généralisé j, qui représente le corps composé des corps $j=1, \ldots n$ :

$$
\begin{gathered}
{ }^{j} J_{j}^{+}={ }^{j} J_{j}+{ }^{j+1} T_{j}^{\mathrm{T}}{ }^{j+1} J_{j+1}^{+}{ }^{j+1} T_{j} \\
{ }^{j} \boldsymbol{\beta}_{j}^{+}={ }^{j} \boldsymbol{\beta}_{j}+{ }^{j+1} T_{j}^{\mathrm{T}}{ }^{j+1} \boldsymbol{\beta}_{j+1}^{+}+{ }^{j+1} T_{j}^{\mathrm{T}}{ }^{j+1} J_{j+1}^{+}{ }^{j+1} \gamma_{j+1}
\end{gathered}
$$

avec :

$$
{ }^{j} J_{j}=\left[\begin{array}{cc}
M_{j} \boldsymbol{I}_{3} & -{ }^{j} \boldsymbol{M} \hat{\boldsymbol{S}}_{j} \\
{ }^{j} \boldsymbol{M} \hat{\boldsymbol{S}}_{j} & { }^{j} \boldsymbol{J}_{j}
\end{array}\right]
$$

$M_{j}$ est la masse du corps $j$. On initialise cette récurrence par: ${ }^{n} J_{n}^{+}={ }^{n} J_{n},{ }^{n} \boldsymbol{\beta}_{n}^{+}={ }^{n} \boldsymbol{\beta}_{n}$.

Finalement l'accélération de la tête se calcule par :

$$
\dot{V}_{o}=-\left({ }^{o} J_{o}^{+}\right)^{-1 o} \boldsymbol{\beta}_{o}^{+}
$$

iii) Récurrence avant pour $j=1, \ldots n$ : 
Dans cette récurrence, on calcule les efforts exercés par le corps j-1 sur le corps j, noté ${ }^{j} \boldsymbol{f}_{j}$, et le couple du moteur $\mathrm{j}$ en utilisant les équations suivantes :

$$
\dot{V}_{j}={ }_{j}^{j} T_{j-1}{ }_{j-1}^{j-l} \dot{V}_{j-1}+{ }^{j} \boldsymbol{\gamma}_{j}, \quad{ }^{j} F_{j}=\left[\begin{array}{c}
{ }^{j} \boldsymbol{f}_{j} \\
{ }^{j} \boldsymbol{m}_{j}
\end{array}\right]={ }^{j} J_{j}^{+j} \dot{V}_{j}+{ }^{j} \boldsymbol{\beta}_{j}^{+}, \quad \Gamma_{j}={ }^{j} F_{j}{ }^{T}{ }^{j} A_{j}
$$

Ce modèle a été simulé en 3D [12] après avoir pris en compte les forces de contact (§6.1). Le système simulé est composé de 12 modules, donc 36 articulations rotoïdes. Les résultats de simulations ont une fois comparées à ceux livrés par le modèle macro-continu, ont été utilisés pour dimensionner les actionneurs du prototype.

\subsection{Modèlisation « poly-articulée hybride »}

Dans ce cas le robot anguille est composé de $n$ modules parallèles connectés en série. La plate forme de chaque module parallèle comporte trois degrés de liberté en rotation par rapport à sa base. Cette structure hybride puisqu'à la fois sérielle et parallèle, représente celle retenue pour le prototype (§ 3.1). Sur la base de ses modèles géométrique et cinématique, la modélisation cinématique du robot est réalisée par des formules récursives généralisant celles des robots série. La dynamique de cette structure hybride est fondée sur l'utilisation de résultats de modélisation de l'équipe robotique de I'IRCCyN dans d'autres projets ROBEA, à savoir projet MAX et projet MP2. Dans ces projets nous avons développé la dynamique d'un robot parallèle [13], ainsi que la modélisation d'un robot hybride général avec une base fixe. Pour le robot anguille, on doit aussi tenir compte du caractère mobil de la base (ici assimilée à la tête). L'algorithme série a été généralisé pour ce cas en utilisant la forme explicite du modèle dynamique d'un seul module. C'est ainsi que nous disposons actuellement des modèles dynamique inverse et direct du prototype (hybride). Ces algorithmes sont fondés sur l'algorithme récursif de Newton-Euler. L'algorithme général a été simplifié afin de négliger les inerties des biellettes (§3.2) devant celles des vertèbres (plate-formes). Cet algorithme est actuellement en cours d'implantation sous Matlab-Simulink. Ile devrait permettre d'étudier la flottabilité et la commande du robot avec un degré de fidélité inaccessible a ses deux concurrents.

\section{Modélisation du contact fluide-structure}

Acteurs : A. Leroyer, M. Visonneau, G. Delhommeau (LMF), F. Boyer, M. Porez (IRCCyN)

\subsection{Introduction}

Comme annoncé, la modélisation du contact fluide-structure procède également selon trois niveaux hiérarchiques du plus simple au plus sophistiqué. Avant d'entre dans les détails de ces trois niveaux, il est important de noter que contrairement à la modélisation solide, celle du fluide et son influence sur la nage de l'anguille ne peuvent trouver de solution aussi « satisfaisante ». En effet, l'anguille comme tout autre poisson utilise activement les phénomènes instationnaires, tels que les vortex 
qu'elle lâche dans son sillage de manière à «optimiser » le rendement de sa propulsion. Or les modèles et simulateurs associés à ces problèmes d'optimisation de la propulsion d'un corps déformable plongé dans un fluide sont à ce jour un sujet émergeant de la mécanique des fluides. Voici à présent les trois niveaux de la modélisation du contact et leur fonctions dans le projet :

$\left.1^{\circ}\right)$ Modèles stationnaire : ceux sont des modèles explicites simplifiés dédiés à la commande et simulation rapide.

$2^{\circ}$ ) Modèle de type fluide parfait: Ils assurent le pont entre les niveaux 1 et 3 et rendent compte de l'influence des lâchés de vortex sur la nage (phénomènes de synchronisation...etc..)

$3^{\circ}$ ) Modèle de type Navier-Stokes : ceux sont les modèles les plus fidèles à la réalité du fluide. Ils constituent le socle de référence de toute tentative de modélisation simplifiée du contact fluide-structure.

\subsection{Modèles stationnaires}

Les modèles de contact dits stationnaires ne tiennent pas compte de l'évolution de l'écoulement autour de l'anguille. Ils sont de ce fait uniquement dépendant du mouvement relatif des sections par rapport au fluide, et, de par leur simplicité constituent une alternative pertinente pour les besoins de la commande et de la simulation rapide. La modélisation Cosserat (\$4.1) fournit un cadre naturel à l'établissement de ces modèles. En effet, tout champ de forces extérieures doit dans cette théorie être modélisé par un champ de torseur appliqué le long de la poutre (section par section). Aussi, avons nous tout d'abord établi un modèle de ces torseurs tenant compte des forces et couples (de roulis) de traînée ainsi que des forces et couples (de roulis) induit par des masses et inerties ajoutées. Au bout du compte, le modèle stationnaire du contact apparaît comme une extension du modèle de Morison des câbles sous marins déformables, ici, au cas d'un câble de section elliptique [14]. Finalement, il est important de noter que la loi de contact est écrite dans les repères mobiles des sections et qu'en tant que telle elle ne dépend que des propriétés du fluide, du Reynolds, et de la géométrie elliptique. Néanmoins, la résultante de ces torseurs (section / section) est ensuite transportée dans le repère tête, intégrant ainsi l'influence de la déformée globale du corps dans le modèle final.

\subsection{Modèles de type fluide parfait}

Ce second modèle vise essentiellement à établir un pont entre le modèle stationnaire et le modèle le plus raffiné du fluide donné par les équations de Navier-Stokes. Le contact est dans ce cas instationnaire et donc déduit de l'intégration numérique de l'écoulement autour du robot. Afin de limiter les temps de calcul au maximum, un modèle de type fluide parfait incompressible est adopté. Dans ces conditions, le champ de vitesse de l'écoulement est régi par l'équation de Laplace de son potentiel sous les conditions aux limites de non pénétration du fluide dans le solide et le repos du fluide à l'infini (ce que nous supposons). Les hypothèses du fluide parfait permettent d'intégrer numériquement l'écoulement à partir d'une méthode de type « intégrale de frontière ». Dans cette méthode, des fonctions harmoniques singulières sont placées sur les frontières et calculées à chaque pas de temps afin 
de satisfaire les conditions limites ainsi que diverses conditions liées au sillage tourbillonnaire de l'anguille : telles que celle de Kutta ou le théorème de Kelvin. Ces modèles, actuellement en cours de développement seront mis à contribution afin d'identifier nos modèles stationnaires après qu'ils aient été eux même recalés par les modèles de type Navier Stokes. Enfin, ils serviront à étudier l'influence des lâchés de vortex (Figure 14), phénomène essentiel à l'étude de la nage.

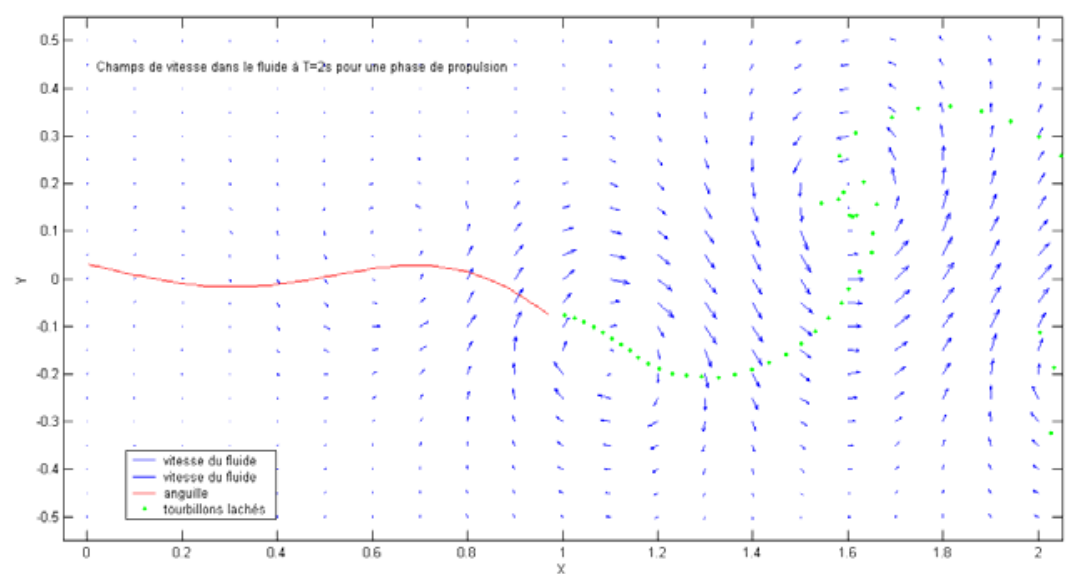

Figure 14 - $\quad$ Modélisation en fluide parfait par la méthode des vortex

Cette dernières figures révèle les lâchés de vortex dans le sillage de l'anguille. Ces vortex lâchés alternativement sont de circulation opposées et réalisent ainsi une allée de Vor-Karman. Cette allée génère un jet en arrière qui par réaction propulse l'animal en avant.

\subsection{Modèles complets : équations de Navier-Stokes}

Les simulations par résolution des équations de Navier-Stokes ont pour objectif essentiel de fournir une base de données de référence afin de valider les modèles simplifiés exposés précédemment. Les calculs sont effectués avec le code ISIS de l'Equipe Modélisation Numérique du LMF. Basé sur une discrétisation fortement conservative de type Volumes-Finis non-structurés avec un nombre de faces arbitraire, ce code résout les équations de Navier-Stokes en moyenne de Reynolds, et peut traiter des écoulements à forts Reynolds sur des configurations géométriques complexes. Pour des écoulements de fluides newtoniens incompressibles, les équations à résoudre s'écrivent :

Conservation de la masse :

$$
\operatorname{div}(u)=0
$$

Conservation de la quantité de mouvement :

$$
\frac{\partial \rho u}{\partial t}+\operatorname{div}(\rho u \otimes u)=\rho g-\operatorname{grad}(p)+\operatorname{div}\left(\overline{\overline{\tau_{v}}}+\overline{\overline{\tau_{t}}}\right)
$$

$u$ : vitesse du fluide en moyenne de Reynolds

$\rho$ : masse volumique du fluide

$p$ : pression du fluide 
g : gravité

$\overline{\overline{\tau_{v}}}=2 \mu \overline{\bar{D}}$ : tenseur des contraintes visqueuses

$\mu$ : viscosité dynamique

$\overline{\bar{D}}$ : tenseur des taux de déformation

$=$

$\tau_{t}:$ tenseur de Reynolds (contraintes turbulentes)

Le champ de vitesse est obtenu à partir des équations de conservation de quantités de mouvement et la pression est déterminée selon la contrainte d'incompressibilité transformée en équation de pression. Les intégrales de volumes et de surfaces sont évaluées par des approximations du second ordre. La discrétisation temporelle est assurée par une méthode du deuxième ordre décentrée à deux pas de temps. Les calculs multiprocesseurs sont gérés par MPI. Différents modèles de turbulence (loi de paroi et proche paroi) sont disponibles, allant de modèles basés sur le concept de viscosité turbulente (fermetures à une ou deux équations) jusqu'à des fermetures au second ordre, reposant sur la résolution des équations de transport des contraintes de Reynolds.

Au cours du projet A. Leroyer [15], a développé des méthodologies (résolution de la dynamique des mouvements d'ensemble rigides d'un corps déformable, techniques de remaillage, couplage écoulement-mouvement,...) qui ont été intégrées au code. Ces nouvelles fonctionnalités permettent de simuler les mouvements 3D d'un nombre quelconque de corps indéformables mais aussi déformables à déformation imposée. La simulation du comportement dynamique d'un robot autopropulsé est ainsi rendue possible. Le mouvement du robot est vu comme la superposition d'un mouvement d'ensemble de nature rigide et d'un mouvement de déformation. La forme du corps est imposée par une loi de courbure déduite de la modélisation macro-continue (§4.1). Ceci nécessite à chaque pas de temps d'adapter le maillage à cette nouvelle forme. Un changement de repère permet ensuite tout au long de la résolution couplée fluide-structure de positionner ce maillage (et donc le robot) dans l'espace. Les paramètres de ce déplacement en bloc sont issus de l'intégration de la dynamique d'ensemble du corps soumis à déformation imposée. Le déplacement du robot est donc uniquement régi par la réponse du fluide à la déformation imposée au corps.

La modification du maillage au cours du temps est prise en compte dans les équations du fluide par l'intermédiaire des flux de vitesse de déplacement du maillage : le point de vue n'est alors plus strictement eulérien, les variables étant attachées au maillage mobile. Le couplage est schématisé par la figure 1 :

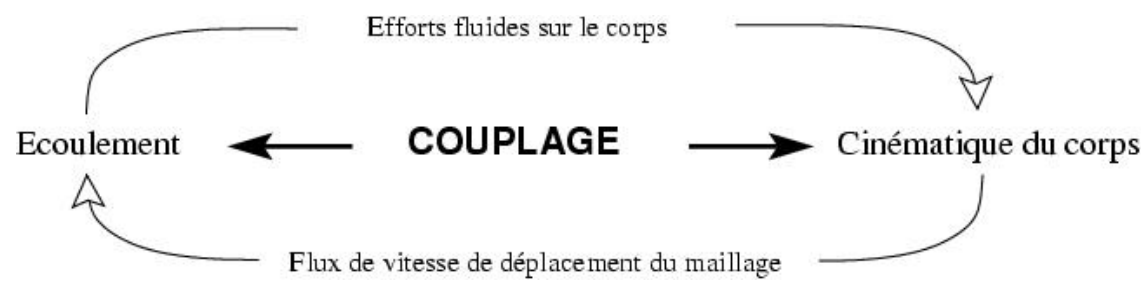

Figure 1. Schématisation du couplage écoulement/mouvement

Pour obtenir un couplage stable, il est nécessaire, d'une part de remettre à jour 
l'interaction écoulement-mouvement au cours de la résolution sur un pas de temps, d'autre part d'effectuer un traitement spécifique des équations de la dynamique d'ensemble (rigide) qui jouent un rôle de sous-relaxation.

Ainsi, sur la base des allures bio-mimétiques (voir II), les premiers calculs tridimensionnels recensés à notre connaissance reproduisant le mécanisme de nage par résolution couplée des équations des Navier-Stokes en moyenne de Reynolds et de la dynamique rigide d'ensemble d'un corps soumis à des déformations imposées, ont été réalisés (Fig. 2).

Pour le moment, seuls des calculs préliminaires sur une géométrie du robot non encore finalisée ont été effectués. Ces derniers ont utilisé une loi de déformation correspondant à un mouvement d'avance en ligne droite mais aussi à un mouvement de virage. Pour ces premières simulations, seuls des mouvements plans ont été considérés. Le domaine de calcul a donc avantageusement été limité au demiespace supérieur avec une condition de symétrie sur le plan $z=0$ (Fig. 3). Deux maillages ont été réalisés sur cette forme temporaire. Le premier, de 65000 cellules, est un maillage adapté à une modélisation de la turbulence de type loi de paroi. Le deuxième, de 120000 cellules, est dérivé du précédent. II a été raffiné près du corps pour appliquer des modèles de turbulence proche paroi. L'influence du modèle de turbulence a été étudiée en examinant les différences obtenues sur la vitesse moyenne limite atteinte pour la loi d'avance périodique avec différents modèles de fermeture (Spalart-Allmaras, $k-\omega, k-\omega S S T, k-\omega B S L$ ) dans les configurations proche paroi et loi de paroi. Deux calculs complémentaires ont été testés : le premier en considérant un écoulement laminaire avec une condition d'adhérence sur le corps, le deuxième avec une condition de glissement sur le corps. II ressort de cette étude une influence assez limitée du modèle de turbulence sur la valeur de la vitesse limite obtenue (l'écart maximal entre les différents résultats étant de l'ordre de 5\%). En revanche, comme on pouvait s'y attendre (voir Fig. 4), le calcul laminaire surestime la vitesse de l'ordre de $25 \%$ par rapport aux vitesses obtenues avec un écoulement turbulent. Enfin, le calcul sous la condition de glissement sur le corps (i.e. sans viscosité) donne une vitesse limite encore supérieure (environ 50\%).

La suite va maintenant consister, une fois la géométrie du robot finalisée, à réaliser de nouveaux maillages et établir une base de données pour différents scénarios particuliers (loi d'avance en étudiant l'influence des paramètres d'amplitude et de fréquence, loi de virage, déformations tridimensionnelles du corps, plongée, influence des nageoires caudales,...). Ils serviront alors de référence pour caractériser et identifier les modèles simplifiés. Qui plus est, une étude énergétique a d'ores et déjà été initiée, pour explorer la notion de rendement. Pour l'heure, le rendement de ce "poisson numérique" est à n'en pas douter plus faible que ceux rencontrés dans la Nature. Dans ce sens, des calculs d'optimisation sur les paramètres du mouvement sont envisagés afin d'améliorer son efficacité. L'un des intérêts de ce genre de simulations est d'avoir accès à des quantités difficilement mesurables au réel. Ceci devrait permettre à terme de mieux comprendre les phénomènes physiques nécessaires pour reproduire les performances aquatiques des poissons. 


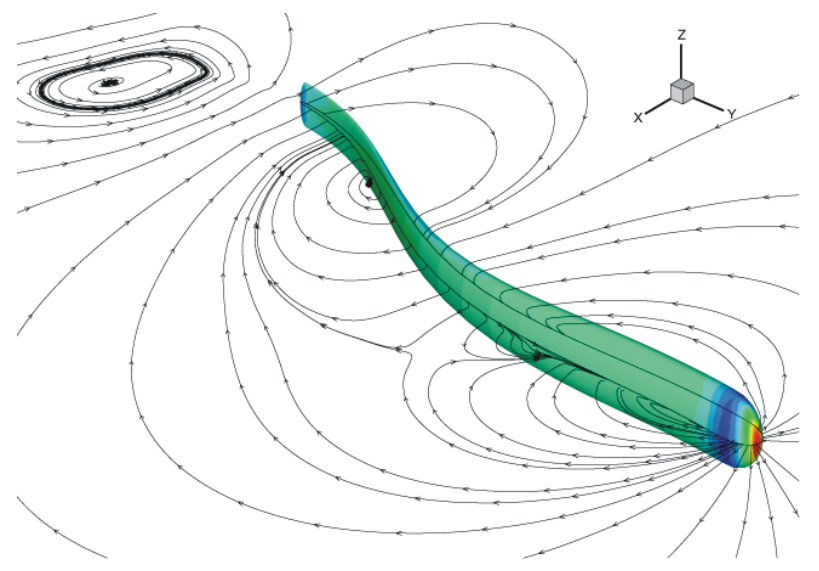

Figure 15 - Visualisation du champ de pression et des lignes de courant

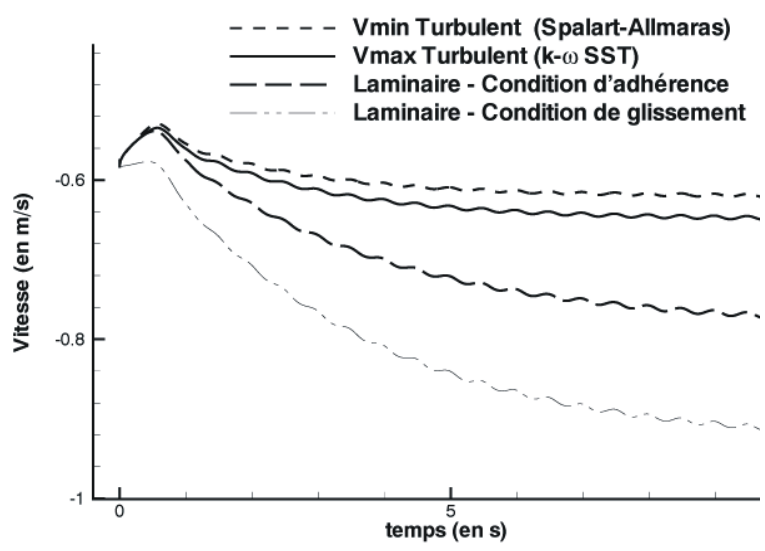

Figure 16 - Évolution de la vitesse en fonction de la modélisation retenue

\section{Commande}

Acteurs : M. Alamir, N. Marchand (LAG), B. Jouvencel, L. Lapierre (LIRRM)

\subsection{Introduction}

Dans cette section (qui reprend les notations des paragraphes $4.1,2,3$ ), les travaux concernant la commande du prototype sont décrits. Ces travaux s'appuient pour l'heure sur le modèle macro-continu (\$4.1) et seront transférés à terme sur le modèle hybride du prototype (§4.6), et in fine, sur le prototype lui même. Même si les choix présentés ici ne sont pas encore définitifs, une bonne vision de l'ensemble émerge. La commande de l'anguille doit permettre de maîtriser les objectifs (ou allures) suivantes :

- Orientation et vitesse dans le plan horizontal.

- Changement d'altitude.

- Maintien d'une bonne posture en roulis.

Ces objectifs doivent être réalisées en utilisant les commandes disponibles, à savoir :

- Les lois de courbure et torsion (vecteur $K_{d}$ )

- Les ailerons (nageoires pectorales) placés au niveau de la tête.

Dans cette présentation et par souci de simplicité, on suppose que les profils de courbure ainsi que l'incidence des ailerons sont parfaitement commandés de telle sorte que leurs valeurs désirées soient instantanément réalisées.

\subsection{Manœuvres dans le plan horizontal $(x, y)$}

Le mouvement de l'anguille dans le plan $(x, y)$ est dû aux ondulations de courbure de son corps dans le plan du lacet, i.e. : autour de l'axe $t_{3}$ de ses sections (lorsque l'anguille est stabilisé en roulis). Ces ondulations sont commandées par la courbure $K_{3}(t, X, s)$, fixée pour réaliser l'ondulation souhaitée. Dans les résultats présentés ici, et sur la base de l'étude bio-mécanique préliminaire (§ 2), la loi de courbure en lacet 
suivante a été adoptée :

$$
K_{3}(t, X, s)=C_{a}(X, s) \sin (2 \pi(X / \lambda+t / T))+\delta_{3}
$$

avec :

$$
C_{a}(t, X, s):=\left\{\begin{array}{c}
C_{a}^{o} \sqrt{\frac{X}{L}}, \text { si }: s=+1 \\
C_{a}^{o} \sqrt{\frac{L-X}{L}}, \text { si }: s=-1
\end{array}\right.
$$

Noter qu'il s'agit d'ondulations d'une période $T$ et d'une longueur d'onde $\lambda$ et dont l'amplitude est fonction de l'abscisse $X$ de la section considérée. Le paramètre $s$ dans les équations ci-dessus définit le sens de l'accélération désirée, à savoir $(s=+1)$ pour le sens direct et $(s=-1)$ pour le sens inverse. Le terme $\delta_{3}$ représente une courbure additionnelle constante le long du corps de l'anguille. En fixant les grandeurs $\lambda$ et $T$, l'entrée de commande pour le mouvement plan est donnée par :

$$
\left(\begin{array}{lll}
C_{o}^{a} & \delta_{3} & s
\end{array}\right)^{T} \in\left[0, C_{a}^{o, \max }\right] \times\left[-\delta_{3}^{\max },+\delta_{3}^{\max }\right] \times\{-1,+1\}
$$

Plus précisément, la première variable $\left(C_{a}^{o}\right)$ contrôle l'accélération asymptotique de la tête de l'anguille alors que la seconde $\left(\delta_{3}\right)$ contrôle l'orientation de la vitesse de celle-ci. En fait, les simulations de tous les modèles développés lors de ce projet montrent que lorsque $\delta_{3}$ est nul, l'anguille adopte un mouvement rectiligne (après un court transitoire courbe) alors qu'une valeur constante de $\delta_{3}$ conduit à un mouvement circulaire.

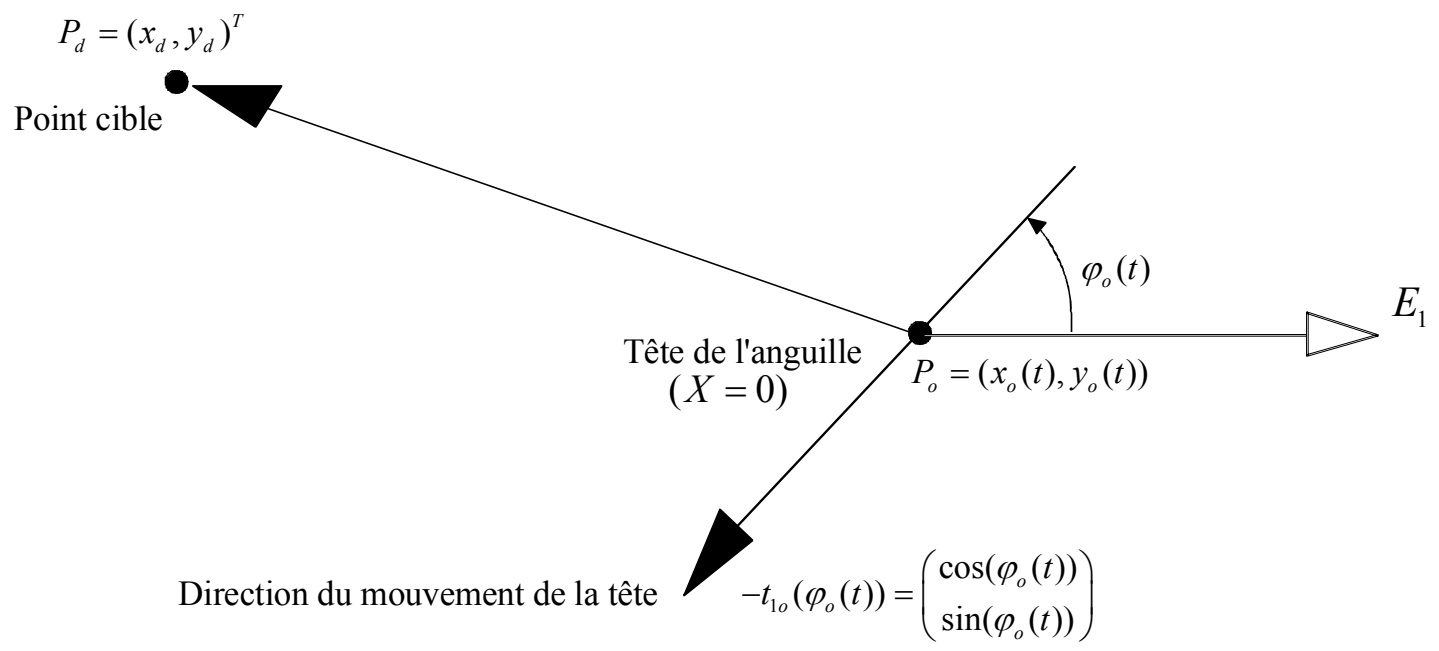

Figure 17 - Schéma de définition des grandeurs invoquées dans la définition de la commande en orientation dans le plan $(x, y)$.

Ceci suggère la commande suivante pour l'orientation dans le plan $(x, y)$ : 


$$
\delta_{3}:=\left\{\begin{array}{c}
\beta \pi_{3}\left(t_{1 o d} \times t_{1 o}\right), \text { si }: t_{1 o d} \cdot t_{1 o} \leq 0 \\
\delta_{3}^{\max }
\end{array}\right.
$$

où $\pi_{3}(v)$ représente la troisième composante du vecteur $v$ et $t_{1 o d}=P_{o} P_{d} /\left\|P_{o} P_{d}\right\|$ représente le vecteur unitaire du cap attendu. II s'agit donc simplement d'un contrôleur proportionnel sur l'indicateur d'alignement entre la vitesse de la tête et la direction souhaitée avec saturation due à une courbure maximale admissible. II est à noter que les expressions ci-dessus sont calculées en considérant les valeurs moyennes de la position et de la vitesse de la tête de l'anguille sur une période d'ondulation $T$.
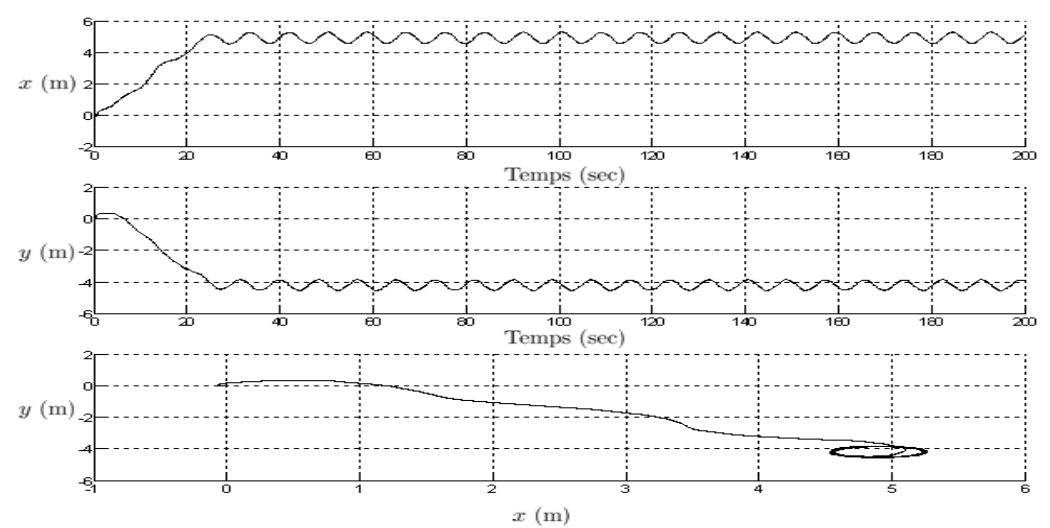

Figure 18 - Évolution des trajectoires moyennes de la tête de l'anguille dans le plan $(x, y)$ avec un point cible $(5,-4)$ et sans contrôle de vitesse.

La figure 17 montre le comportement de l'anguille sous l'effet de la loi de commande décrite ci-dessus lorsqu'un point $(5,-4)$ est pris comme cible et en l'absence de contrôle de la vitesse. L'anguille s'oriente automatiquement vers le point cible et « lui tourne autour».

\subsection{Régulation de la vitesse dans le plan $(x, y)$}

Afin de contrôler la vitesse de l'anguille, le simulateur est d'abord utilisé pour identifier un modèle dynamique de celle-ci. Le modèle ainsi obtenu présente la structure suivante :

$$
\dot{V}=-\left[\beta_{1} C_{a}^{o}+\beta_{o}\right]\left[V-s V_{\infty}\left(C_{a}^{o}\right)\right]
$$

où $V$ est la vitesse d'avance dans le sens du cap et les paramètres $\left(\beta_{i}\right)$ sont obtenus par identification. II est à noter que ce modèle présente une vitesse limite dont le sens dépend du signe de la variable $(s)$ et un temps de réponse qui dépend du facteur multiplicatif qui est fonction de l'amplitude de l'oscillation définie par $C_{a}^{o}$.

L'équation précédente est alors utilisée pour trouver les paramètres $\left(s, C_{a}^{o}\right)$ qui permettent d'imposer à la vitesse une loi de régulation du premier ordre donnée par :

$$
\dot{V}=-\lambda_{V}\left(V-V_{d}\right) ; \lambda_{V}>0
$$


où $V_{d}$ est la vitesse désirée. Les paramètres permettant d'effectuer la poursuite sont alors obtenus en résolvant le problème d'optimisation contraint suivant

$$
\min _{\left(s, C_{a}^{o}\right) \in\{-1,1\} \times\left[0, C_{a}^{\max }\right]}\left|\frac{\lambda_{V}\left(V-V_{d}\right)}{\beta_{1} C_{a}^{o}+\beta_{o}}+s\left[\alpha_{1} C_{a}^{o}+\alpha_{2}\left[C_{a}^{o}\right]^{2}\right]-V\right|
$$

qui équivaut à deux problèmes d'optimisation scalaires sur une intervalle bornée $\left[-C_{a}^{o, \max }, C_{a}^{o, \max }\right]$. Là encore, les variables apparaissant dans les formules ci-dessus sont les moyennes des variables sur une période d'ondulation.

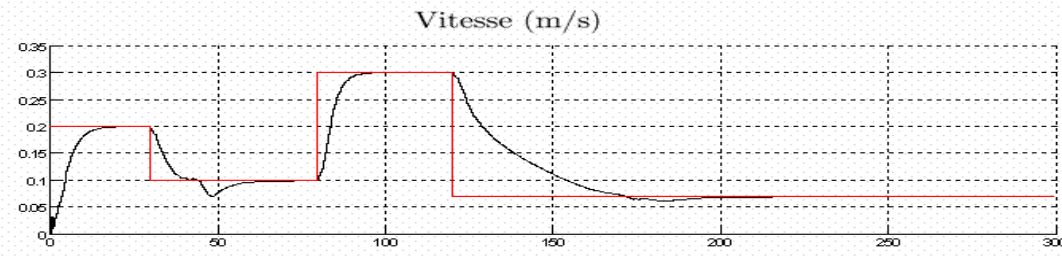

La commande $C_{0}^{0}$

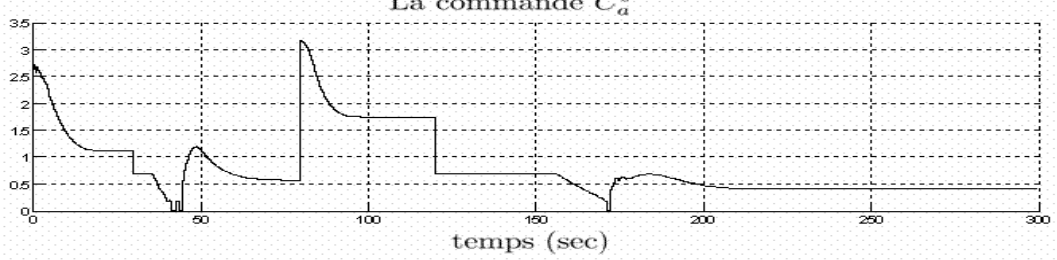

Figure 19 - Évolution de la vitesse moyenne de la tête de l'anguille pour différents échelons de consigne et la commande associée.

La figure 18 montre le comportement du système en boucle fermée face à un changement de consigne de la vitesse.

\subsection{Manœuvre de changement d'altitude $z$}

Le changement d'altitude se fait essentiellement grâce aux forces hydrodynamiques provoquées par la présence des ailerons au niveau de la tête de l'anguille. Afin d'aboutir à une commande peu sensible à la modélisation, une approche simple a été adoptée dans laquelle, le mouvement du point d'appui des ailerons a été modélisé au moyen d'une force inconnue $R$ (Figure 19) provenant du mouvement de la partie arrière du corps de l'anguille. L'idée est alors de développer une commande de changement d'altitude qui estime partiellement cette force ou la compense d'une façon robuste sur la base d'une borne supérieure de l'amplitude de la composante de $R$ selon l'axe $z$. 


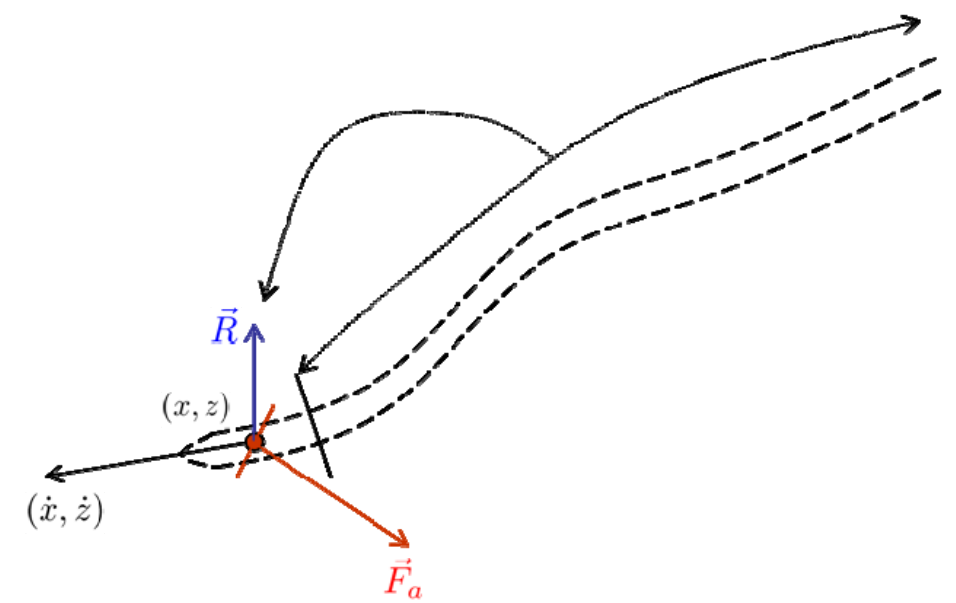

Figure 20 - Schéma de principe de la commande de changement d'altitude.

L'équation en $z$ du point d'appui s'écrit

$$
\ddot{z}=F_{a, z}(\alpha, \dot{x}, \dot{z})+R_{2}
$$

où $R_{2}$ est la composante selon $z$ de la force inconnue et

$$
F_{a, z}=-\mu_{a} \sqrt{\dot{x}^{2}+\dot{z}^{2}} \cos (\alpha)[-\dot{x} \sin \alpha+\dot{z} \cos \alpha]
$$

est la composante selon $z$ de la force hydrodynamique due aux ailerons faisant tout deux l'angle $\alpha \in\left[-\alpha_{\max }, \alpha_{\max }\right]$ par rapport à la direction de la vitesse moyenne de la tête. L'angle des ailerons est alors pris comme la solution du problème d'optimisation suivant :

$$
\hat{\alpha}\left(z, \dot{z}, \dot{x}, z_{d}\right)=\operatorname{Arg} \min _{\alpha \in\left[-\alpha^{\max }, \alpha^{\max }\right]}\left|F_{a, z}(\alpha, \dot{x}, \dot{z})-u_{2}^{d}\left(z, \dot{z}, z_{d}\right)\right|
$$

de manière à ce que la composante verticale de la force hydrodynamique soit la plus proche possible de la valeur:

$$
u_{2}^{d}\left(z, \dot{z}, z_{d}\right)=\left\{\begin{array}{l}
-\lambda_{3} \dot{z}-\hat{R}_{2}-\mu_{s} S\left(z, \dot{z}, z_{d}\right): \text { version adaptative } \\
-\lambda_{3} \dot{z}-\left[R_{2}^{\max }+\mu_{s}\right] \operatorname{Sign}_{\varepsilon}(S): \text { version robuste }
\end{array}\right.
$$
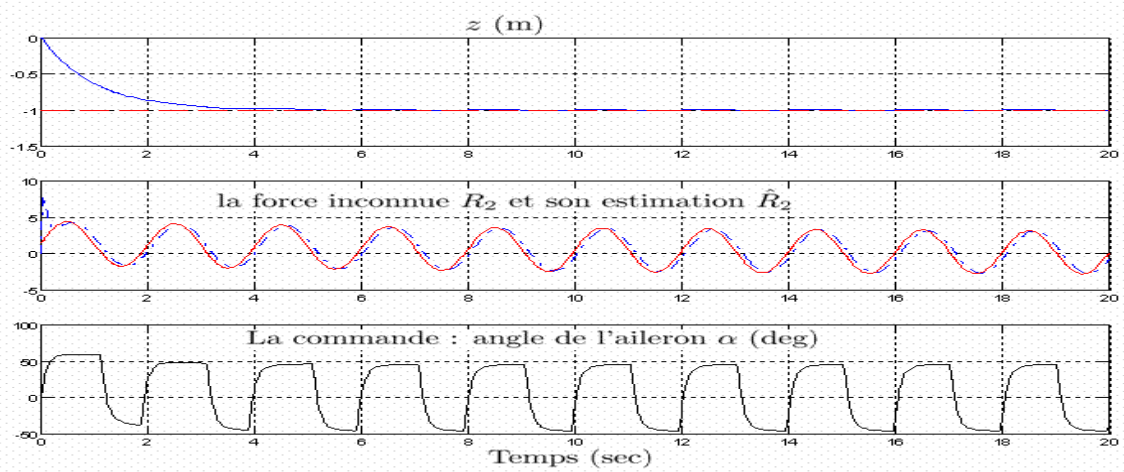

Figure 21 - Scénario de changement d'altitude (échelon négatif de $1 \mathrm{~m}$ ) sous la version adaptative du contrôleur proposé. La valeur maximale de l'angle de l'aileron admissible est de 60 deg. La commande rejette une perturbation arbitraire synchrone avec la loi de courbure. 
où la fonction $S$ est une surface stabilisante définie par

$$
S\left(z, \dot{z}, z_{d}\right)=\dot{z}+\lambda_{3}\left(z-z_{d}\right)
$$

$\hat{R}_{2}$ dans la version adaptative est une estimation de $R_{2}$ obtenue à travers la loi d'adaptation suivante :

$$
\dot{\hat{R}}_{2}=\frac{1}{\beta} S\left(z, \dot{z}, z_{d}\right)
$$

alors que dans la version robuste $R_{2}^{\max }$ représente une borne supérieure connue de la valeur absolue de $R_{2}$. Finalement, la fonction $\operatorname{Sign}_{\varepsilon}($.$) est une version régularisée$ de la fonction signe alors que $\lambda_{3}, \beta$ et $\mu_{s}$ sont des réel positifs convenablement choisis.

La figure 20 montre un scénario de changement d'altitude suite à un échelon de $-1 \mathrm{~m}$ dans le cas où la version adaptative est utilisée. La deuxième courbe montre la valeur réelle et estimée de la force (non mesurée et de moyenne non nulle) due au mouvement global de l'anguille.

La commande de stabilisation en roulis se fait de la même manière en considérant la différentielle d'angle entre les deux ailerons droit et gauche et en suivant les mêmes lignes directrices que celles adoptées pour la manœuvre de changement d'altitude.

\section{Implémentation informatique}

\section{Acteurs : J.L. Bechennec, P. Lemoine, Y. Trinquet, S. Bellavoire (IRCCyN)}

\subsection{Contraintes technologiques}

Pour ce type de projet où les systèmes d'actionnement et de commande sont embarqués, les notions d'encombrement, de poids et de consommation d'énergie sont essentielles. Un autre aspect est la partie câblage du système. Il faut éviter en effet que les divers câbles d'alimentation et de transport d'informations ne viennent réduire la capacité de déformation de l'anguille par l'introduction de couples ou de forces supplémentaires à vaincre au cours des mouvements.

Pour réaliser les déformations du corps de l'anguille, chaque articulation est actionnée par trois moteurs localisés dans une des vertèbres adjacentes (cf. §3.2). L'ensemble des moteurs est donc réparti sur toute la longueur de l'anguille. Cette architecture permet d'obtenir une répartition naturelle des masses le long du corps de l'anguille, contrairement à une solution du type actionnement par tringles ou câbles avec une concentration des actionneurs en un point du robot. Le prototype comporte 12 articulations actionnées, ce qui porte à 36 le nombre de moteurs nécessaires à sa déformation.

Pendant la phase de conception il a fallu veiller à intégrer toutes ces notions de sorte à minimiser:

- les volumes d'occupation des actionneurs et des systèmes électroniques et informatiques de commande, afin de laisser libre cours à une amplitude maximale de déformation du corps de l'anguille, 
- le nombre et la section des fils conducteurs d'alimentation et de transport d'informations, de sorte à obtenir un faisceau de câblage qui perturbe le moins possible les mouvements de déformation,

- la consommation électrique globale, de sorte à assurer une autonomie en énergie suffisante,

- la surcharge pondérale liée aux éléments électriques et électroniques, de sorte à ne pas pénaliser la flottabilité de l'ensemble du robot.

Enfin il a fallu également veiller à ce que les divers composants soient répartis de manière à assurer un bon équilibrage de la masse du robot.

D'autre part, on cherche au final à piloter la tête du robot anguille. En effet c'est sa position, son sens et sa vitesse de déplacement que l'opérateur cherche à commander. La propulsion nécessaire à ces déplacements est générée grâce à la déformation du corps de l'anguille.

On voit donc que toutes les informations relevant du positionnement, de l'orientation ou de l'avancement du robot concernent la tête de l'anguille. II est donc naturel que la tête soit instrumentée avec différents capteurs (inclinomètres, accéléromètres, mesure de vitesse relative ...), et qu'elle soit prise comme référence pour définir les déformations du reste du corps. Même si certaines informations ne sont pas nécessaires pour la commande dans un contexte télé-opéré "à vue", elles peuvent être importantes pour estimer les performances du prototype et pouvoir reconstruire sa trajectoire relativement au milieu environnant, ce qui rend l'utilisation d'un minimum d'instrumentation pertinent.

Bien entendu, le prototype étant télé-opéré, il est nécessaire de mettre en place un canal de communication entre l'anguille et l'opérateur, ce dernier faisant partie intégrante de la boucle de commande en jouant le rôle de donneur d'ordres et de correcteur de trajectoire.

Enfin, il est nécessaire d'effectuer périodiquement un certain nombre de calculs séquentiels pour chaque articulation (calcul des consignes angulaires de chaque moteur et boucles d'asservissement de position). Plus le nombre d'articulations (et de vertèbres) est important et plus le nombre de calculs à effectuer dans un laps de temps donné est important. Cela nous impose des contraintes en terme de puissance de calcul globale du système de commande en fonction du nombre de vertèbres.

\subsection{Solution retenue}

Toutes ces considérations nous ont amenés à opter pour une solution informatique basée sur un ensemble de calculateurs répartis sur les vertèbres et la tête de l'anguille (et dont le nombre dépend du nombre d'articulations motorisées).

Grâce à cette architecture la tête de l'anguille possède son propre calculateur permettant de gérer les différents capteurs, la communication avec l'opérateur et remplissant le rôle de superviseur de l'ensemble du robot. Les autres calculateurs déchargent le superviseur des opérations devant être effectuées périodiquement pour déterminer les consignes à transmettre à chaque moteur et gérer les boucles d'asservissement de position locales correspondantes.

Cette solution permet d'une part de mettre en place un véritable parallélisme d'exécution et fait d'autre part que l'augmentation du nombre de vertèbres est accompagnée d'une augmentation du nombre de calculateurs. La puissance de calcul globale du système s'adapte donc automatiquement au nombre de vertèbres 
de l'anguille.

Pour le support matériel de notre application, notre choix s'est porté sur le module de la société Phytec construit autour du microprocesseur Motorola MPC565 et présenté sur la figure 21. Ce processeur RISC 32 bits de la famille PowerPC MPC500 est cadencé à $40 \mathrm{MHz}$, possède une unité de calcul flottant 64 bits, et offre des possibilités d'interfaçage nous permettant de piloter 6 moteurs (soit 2 articulations) ainsi qu'un certain nombre de fonctions d'entrée/sortie basées sur l'exécution d'un microcode indépendant et en parfait parallélisme par rapport au processeur principal. Tous ces modules d'exécution indépendants ainsi que le processeur principal ont accès à des unités de gestion du temps permettant entre autre de générer des interruptions périodiques et indépendantes pour chaque bloc d'exécution. Enfin le MPC565 intègre la gestion de 3 interfaces bus CAN permettant de transmettre des messages de 8 octets maximum avec un débit de 1Mb/s pour chacun des bus. Ces transmissions sont de type asynchrone multi-maîtres, avec gestion des priorités (le message le plus prioritaire n'est pas détruit en cas de collision).

Pour finir le module Phytec MPC565 affiche une consommation maximale de 2,15W (dont $0,8 \mathrm{~W}$ pour le processeur Motorola), il ne mesure que $84 \times 57 \times 3 \mathrm{~mm}$ (format proche de la carte de crédit) et ne pèse que $27 \mathrm{~g}$. Bien entendu dans notre application nous n'utiliserons qu'un ensemble réduit de ses capacités d'entrée/sortie, ce qui diminuera considérablement la consommation globale des modules.

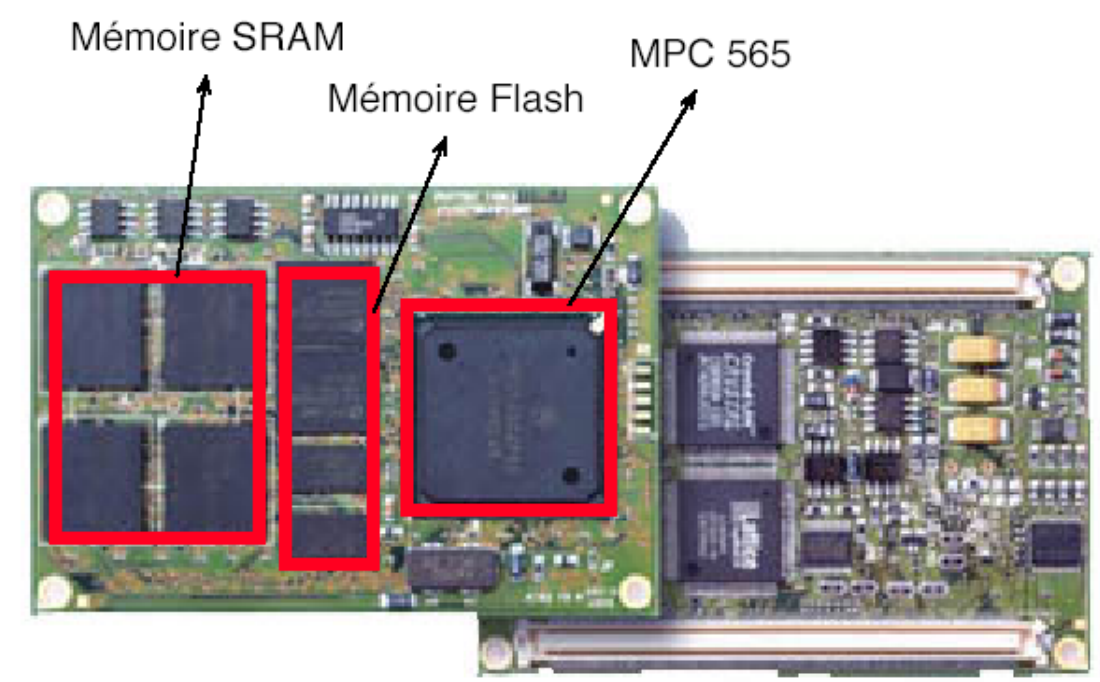

Figure 22 - vues recto et verso du module Phytec MPC565

La possibilité pour chaque processeur MPC565 de pouvoir piloter 6 moteurs nous permet de n'utiliser qu'un seul module pour gérer 2 articulations motorisées. Cette faculté a été mise à profit comme indiqué au §3.3 en alternant les vertèbres supportant les moteurs et celles supportant les modules informatiques et électroniques. Les batteries sont réparties le long de l'anguille en plaçant 4 éléments sur chaque vertèbre support de module électronique/informatique, les autres étant situés dans la tête et la queue. Ceci permet de rééquilibrer les masses le long de l'anguille malgré l'alternance des vertèbres. La figure 22 représente la répartition des modules le long du corps de l'anguille. 


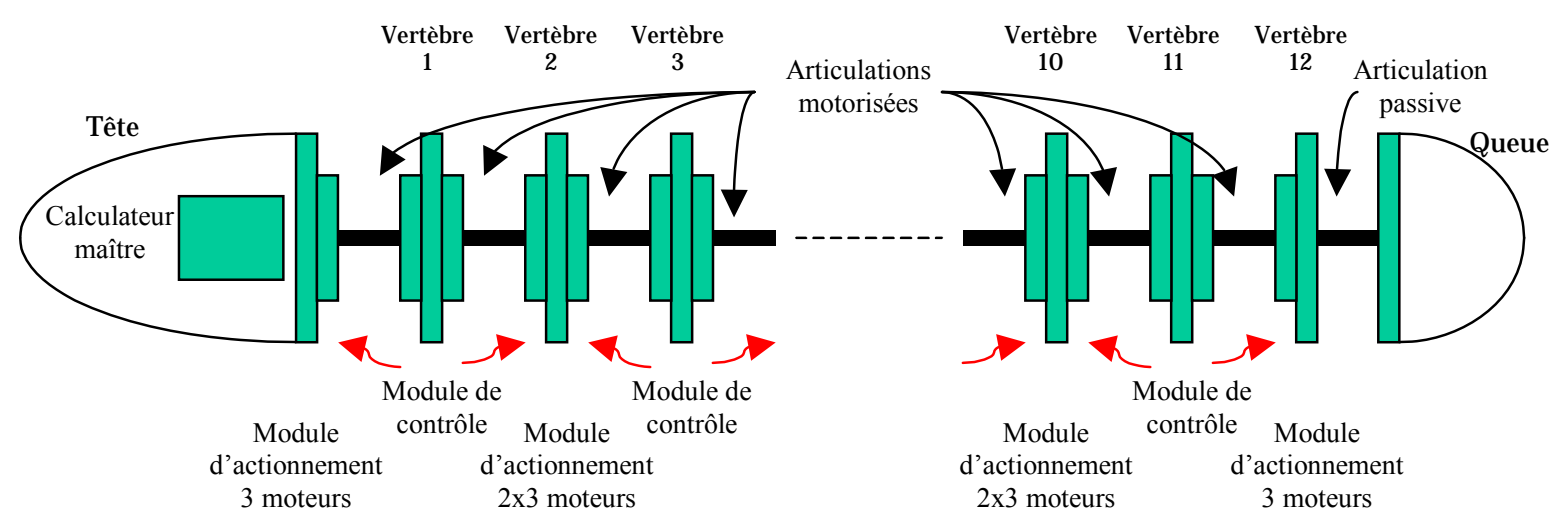

Figure 23 - Répartition des modules le long de l'anguille

Le fait de regrouper les éléments par modules fonctionnels (modules d'actionnement et modules de contrôle) permet de les confiner dans des espaces compacts et bien définis, de réduire le câblage d'interconnexion entre la partie informatique et la partie électronique constituant à elles deux les modules de contrôle, et contribue à limiter le nombre de fils nécessaires pour alimenter et communiquer avec les divers éléments. Pour assurer une plus grande souplesse des fils d'alimentation, leur section a été réduite en alimentant l'ensemble des modules sous une tension de 39,6V (utilisée pour le pilotage des moteurs), réduisant ainsi l'intensité nécessaire, puis en convertissant la tension selon le besoin au plus près des éléments à alimenter.

De plus ce regroupement en modules fonctionnels crée des blocs physiques plus facile à rendre étanche qu'une multitude d'éléments séparés.

L'application logicielle quant à elle s'appuie sur un exécutif temps-réel compatible OSEK-VDX développé par l'équipe Systèmes Temps-Réel de l'IRCCyN. II s'agit d'un système d'exploitation temps-réel multitâches à priorités fixes, intégrant des services de gestion de tâches, de synchronisation, d'exclusion mutuelle, de traitement évènementiel (interruptions, alarmes) et des services de communication s'appuyant sur le bus CAN.

Certaines tâches très spécifiques, comme la gestion des codeurs incrémentaux (capteurs de position des moteurs), sont gérées directement par des fonctions exécutées à l'aide d'un microcode indépendant et parallèlement au processeur principal. Cette possibilité fait partie des fonctions disponibles du MPC565 et décharge le processeur principal de la gestion des nombreuses interruptions liées à ces tâches (2 entrées d'interruption par moteur, soit 12 par module!).

L'application globale est construite sur une architecture répartie, et est constituée d'une tâche de supervision, supportée par le module situé dans la tête de l'anguille, et de tâches de commande locales, supportées par les modules situés dans les vertèbres de l'anguille (1 vertèbre sur 2). Cette vision est très synthétique et nous ne détaillerons pas ici les tâches précédemment citées qui sont en fait constituées chacune de plusieurs tâches concurrentes.

Cependant il est nécessaire de considérer ici brièvement l'aspect communication et synchronisation entre ces diverses tâches telles que définies ci-dessus. En effet la tâche superviseur doit transmettre à l'ensemble des tâches de commande locales les informations correspondant à l'allure courante de l'anguille imposée par l'opérateur. Ces informations concernent les paramètres de l'ondulation devant se propager le long du corps de l'anguille, à laquelle vient se superposer éventuellement une courbure globale. Ces données peuvent être transmises simultanément à toutes les tâches de commande locales au moyen de la diffusion sur le bus CAN d'un message unique. A l'aide de ces informations, chaque tâche de commande locale peut calculer 
les consignes angulaires des 2 articulations qu'elle contrôle, en fonction du temps et de la position des articulations dans la chaîne constituant l'anguille. L'aspect propagation de la déformation le long du corps de l'anguille (notamment lors de l'enchaînement d'allures différentes) est assuré par une synchronisation des tâches de commande entre elles au moyen de messages spécifiques déposés sur le bus CAN à destination de la tâche de commande située sur le module suivant dans la direction de la propagation.

Ces fonctions de synchronisation et de communication s'appuient sur le premier bus CAN. Le deuxième bus CAN est utilisé à des fins de diagnostic et au retour d'informations en provenance des vertèbres vers la tête de l'anguille. Cette solution évite de perturber les fonctions de commande par les fonctions de surveillance et de diagnostic.

\section{Conclusion et perspectives}

Le projet «Robot anguille » soutenu par le programme national ROBEA du CNRS entre à présent dans sa seconde moitié. Parmi les résultats obtenus, citons ceux concernant la conception mécanique qui a abouti, sur la base d'un empilement sériel de modules parallèles, à une première version CATIA du prototype. Reste sur ce point deux problèmes essentiels à résoudre : $1^{\circ}$ ) la peau, pour laquelle, même si des solutions ont été proposées, nombre d'incertitudes subsistent. $2^{\circ}$ ) la flottabilité du prototype, qui devra être approfondie dans l'avenir et déboucher sur des systèmes de ballaste ou vessie... Enfin notons que du point de vue des connaissances, de nombreux résultats ont vu le jour. Parmi ceux ci, citons ceux liés à la mécanique des fluides (Navier-Stokes), à la robotique hyper - redondante (modélisation macrocontinue), à la robotique poly-articulée (extension du formalisme de Newton - Euler à la problématique de la locomotion)... Finalement, une approche réaliste de la commande est en cours de développement et validation sur les simulateurs. Elle devrait permettre à terme de contrôler le prototype en mode télé-opéré et in finé de tester les performance de l'approche bio-mimétique en les comparant à celles obtenues sur des sous-marins plus conventionnels.

\section{Bibliographie}

[1] Merlet, J.P. "Parallel Robots", Kluwer Academic Publishers, 2000.

[2] Karouia, M., "Conception structurale de mecanismes paralleles spheriques", Thèse de doctorat, RI 2003-26, École Centrale de Paris, 2003.

[3] Gosselin, C., Hamel, J.F., "The agile eye: a high performance threedegree-of-freedom camera-orienting device", IEEE Int. conference on Robotics and Automation, pp.781-787. San Diego, 8-13 Mai, 1994.

[4] Birglen, L., Gosselin, C., Pouliot, N., Monsarrat, B., et Laliberté, T., "SHaDe, a new 3-dof haptic device", IEEE Transactions on Robotics and Automation, Vol. 18, No. 2, pp. 166-175, 2002.

[5] Agrawal, S.K, Desmier, G. et S. Li. "Fabrication and analysis of a novel 3 dof parallel wrist mechanism", ASME J. of Mechanical Design, 117(2):343-345, Juin 1995.

[6] Chablat, D. et Wenger, P. : "Design of a Spherical Wrist with Parallel 
Architecture: Application to Vertebrae of an Eel Robot", ICRA05, Barcelone, Avril 2005.

[7] Cosserat E et F., Théorie des corps déformables, Hermann, Paris, 1909.

[8] Chirikjan, G.S., Burdick, J.W., "An obstacle avoidance for hyper-redundant manipulators", IEEE Int. Conf. Robotics and Automation, OH, May, 1990, pp. 14-17.

[9] Simo J.C. and Vu-Quoc L. "A three-dimensional finite-strain rod model. Part II : computational aspects", Comp. Meth. Appl. Mech. Eng., 58, 1986, 79-116.

[10] Simo J.C. and Vu-Quoc L. "On the dynamics in space of rods undergoing large motions - A geometrically exact approach", Comp. Meth. Appl. Mech. Eng., 66, 1988, 125-161.

[11] Luh J.Y.S., Walker M.W., Paul R.C.P., "On-line computational scheme for mechanical manipulator", Trans. of ASME, J. of Dynamic Systems, Measurement, and control, Vol. 102(2), 1980, pp.69-76.

[12] Boyer, F., Porez, M., Khalil, W., "Macro-continuous computed torque algorithm for a three-diemensional eel-like robot", Rapport interne Ecole des Mines de Nantes, soumis à IEEE Trans. On Robotics and Automation, (en révision).

[13] Khalil W., Kleinfinger J.-F., "Minimum operations and minimum parameters of the dynamic model of tree structure robots", IEEE J. of Robotics and Automation, Vol. RA-3(6), December 1987, p. 517-526.

[14] Khalil, W., Gallot, G., Ibrahim, O., Boyer, F., "Dynamic modelling of a 3D serial eel-like robot", ICRA05, Barcelone, Avril 2005.

[15] Khalil, W., Ibrahim, O., "General Solution for the Dynamic Modeling of Parallel Robots", ICRA04, New Orleans, LA, 4, pp.3665-36670.

[16] Burgess, J.J., Bending Stiffness in a simulation of undersea cable deployment, Intern. Journal of Offshore and Polar Engineering, Vol.3, No.3, September 1993.

[17] Leroyer, A., «Etude du couplage écoulement / mouvement pour des corps solides ou à déformation imposée par résolution des équations de Navier-Stokes. Contribution à la modélisation numérique de la cavitation. » ftp://ftp.ec-nantes.fr/pub/DMN/Thesis/these_leroyer.ps.gz, Nantes Université de Nantes, 2004. 
